\title{
Electron momentum-space densities of Li metal: A high-resolution Compton-scattering study
}

\author{
W. Schülke, G. Stutz, F. Wohlert, and A. Kaprolat \\ Institute of Physics, University of Dortmund, D-44221 Dortmund, Germany
}

(Received 19 March 1996)

\begin{abstract}
Directional Compton profiles (CP's) of Li metal were measured for 11 directions of the momentum transfer q with 0.14 a.u. (a.u. $=$ atomic units: $\hbar=e=m=1$ ) momentum-space resolution using synchrotron radiation from the DORIS (Doppel-Ring-Speicheranlage) storage ring monochromatized to $31 \mathrm{keV}$. Both the total valence-electron CP's and the directional differences of the CP's exhibit considerable deviations from the most recent density-functional calculations, performed by Sakurai et al. [Phys. Rev. Lett. 74, 2252 (1995)] within the limits of the local density approximation. These discrepancies are attributed to self-energy effects connected with the excitation of so-called plasmaron modes. The three-dimensional (3D) valence-electron momentum density, $\rho(\mathbf{p})$, as well as the $3 \mathrm{D}$ occupation number density $N(\mathbf{k})$, were reconstructed using the Fourier-Bessel method. The reconstructed $\rho(\mathbf{p})$ exhibits clear evidence of higher momentum components due to 110 umklapp processes. The reconstructed $N(\mathbf{k})$ enables a direct experimental access to the Fermi-surface anisotropy of $\mathrm{Li}$, which was found to be $3.6 \pm 1.1 \%$. The reconstructed $N(\mathbf{k})$ for $\mathbf{k} \|[001]$ was fitted to a model with the renormalization factor $z$ as the only free parameter, which was found to be $z=0.1 \pm 0.1$. [S0163-1829(96)07843-5]
\end{abstract}

\section{INTRODUCTION}

Since early studies by DuMond, ${ }^{1}$ it is well known that the measurement of the spectral distribution of the Dopplerbroadened Compton shift (Compton profiles) yields information about the electron momentum distribution of the scattering system, provided the conditions for the so-called impulse approximation are met, as formulated by Eisenberger and Platzman. ${ }^{2}$ The first pioneering Compton profile measurements (see, e.g., Refs. 1 and 3) were done by using conventional x-ray sources and crystal dispersive analysis, ending up with a momentum space resolution of the order of 0.2 a.u. (a.u. $=$ atomic units: $\hbar=e=m=1$ ), but with relative poor statistical accuracy, so that only investigations on low- $Z$ elements could be performed, for example Compton measurements on single crystal $\mathrm{Li}$ metal. ${ }^{4}$ The improvement of the energy resolution of solid-state detectors enabled measurements of Compton profiles using monochromatic $\gamma$-ray sources with photon energies between 60 and $600 \mathrm{keV}$, first introduced by Eisenberger and Reed. ${ }^{5}$ This way a rather good statistical accuracy could also be obtained for high- $Z$ elements. But even by using high-performance solid-state detectors and very high photon energies, the $\gamma$ Compton technique could not achieve a better momentum resolution than 0.45 a.u. Nevertheless, a large number of systems have been investigated during that era of $\gamma$ Compton scattering, for a review see Ref. 6. Moreover, some steps were done made toward a better understanding of the information content of Compton profiles by considering the properties of their Fourier transforms (so-called reciprocal form factors), ${ }^{7-9}$ and toward a more informative representation of experimental results by utilizing methods for reconstructing the three-dimensional (3D) momentum density ${ }^{10,11}$ and the $3 \mathrm{D}$ occupation number density in Bloch $\mathbf{k}$ space. ${ }^{12,13}$ Only the advent of strong synchrotron radiation sources with photon energies between 20 and $60 \mathrm{keV}$ made feasible the crystal-dispersive energy analysis of the scattered radiation, so that a momentum-space resolution of the order of 0.1 a.u. and, at the same time, a high statistical accuracy by using highly penetrating $\mathrm{x}$ rays could be obtained. ${ }^{14-16}$ This way, details of the electron momentum densities of simple metals and alloys, ${ }^{17-19}$ not accessible in $\gamma$ Compton studies, could be resolved, and were discussed in terms of Fermi-surface features. For Li ${ }^{18}$ the anisotropy of the Fermi surface was determined by looking at the behavior of the second derivative of the directional Compton profiles, although the interpretation of the data needed some support from calculations presented together with measurements. Moreover, highresolution Compton profile measurements on Be (Ref. 17) and Li (Ref. 18) metals have revealed discrepancies, when they were compared with calculations, performed within the limits of the local-density approximation (LDA) and corrected with respect to correlation by using the Lam-Platzman scheme. ${ }^{20}$ These discrepancies can certainly be attributed, at least in part, to inadequacies in treating correlation, when calculating the electron momentum densities and Compton profiles. But part of them could also arise from difficulties with handling background in the course of data processing, or could be removed by going beyond the impulse approximation.

Therefore, we decided to measure independently a set of directional Compton profiles of $\mathrm{Li}$ with 0.14-a.u. momentum-space resolution, in order to attack the following problems: (i) 3D reconstruction of the electron momentumspace density together with corresponding error maps in order to find unambigously location and strength of higher momentum components; (ii) measurement of the Fermi-surface anisotropy, free from any models or any help from theoretical calculations, by using a 3D reconstruction scheme for the occupation number density $N(\mathbf{k})$; (iii) fitting of the reconstructed occupation number density to different models of correlation-induced reduction of the discontinuity of $N(\mathbf{k})$ at the Fermi momentum, in order to test, to what extent current theories of correlation effects on momentum space densities 
can account for the experimental results; and (iv) a search for a semiquantitative explanation for discrepancies between the Compton measurements and LDA Compton profile calculations on the basis of experimental results, by taking into account self-energy effects of the inelastic scattering process, which provides the information on Compton profiles.

One very important reason for choosing Li metal as a test ground was the availability of highly sophisticated LDAbased momentum density calculations on $\mathrm{Li}^{18}{ }^{18}$ which can serve as a reliable reference. According to the program as sketched above, the rest of the paper is organized as follows: In Sec. II the fundamental relations are presented, which are used to find an appropriate interpretation of the Compton data, and to perform both the 3D reconstruction of the electron momentum space densities from a set of directional Compton profiles, and the 3D reconstruction of the occupation number density of the Bloch $\mathbf{k}$ space. In Sec. III the Compton experiment itself together with the various steps of data processing are demonstrated. In Sec. IV the results of $\mathrm{Li}$ Compton profile measurements are shown in the form of total valence Compton profiles and of directional difference Compton profiles, in every case confronted with corresponding LDA calculations. In Sec. V we show the result of the 3D reconstruction of the electron momentum densities together with the corresponding error maps, and in Sec. VI the reconstruction of the occupation number density $N(\mathbf{k})$ in Bloch $\mathbf{k}$ space, together with the determination of the $\mathrm{Li}$ Fermi surface anisotropy as directly deduced from the reconstructed $N(\mathbf{k})$. Section VI offers the results of fitting jellium models of $N(k)$ with varying renormalization constant the experimental $N(\mathbf{k})$. Section VII is devoted to a critical inspection of the Compton-scattering process in terms of selfenergy effects, which offers a semiquantitative explanation of discrepancies between measurements and LDA calculations, and which sheds light on how to proceed with an improved interpretation of Compton data. Finally in Sec. VIII we provide conclusions.

\section{BASIC RELATIONS}

The goal of inelastic x-ray scattering experiments is the measurement of the double-differential scattering cross section (DDSCS) $d^{2} \sigma / d \omega_{2} d \Omega$ for a given momentum transfer

$$
\mathbf{q}=\mathbf{K}_{1}-\mathbf{K}_{2}, \quad|\mathbf{q}| \cong 2\left|\mathbf{K}_{1}\right| \sin (\theta / 2),
$$

where $\mathbf{K}_{1}$ and $\mathbf{K}_{2}$ are the wave vectors, $\omega_{1}$ and $\omega_{2}$ are the frequencies of the incident and the scattered photons, respectively. $\theta$ is the scattering angle. Within the limits of a firstorder nonrelativistic perturbation treatment and by neglecting the $\mathbf{p} \cdot \mathbf{A}$ term (A vector potential operator) in the interaction Hamiltonian, the DDSCS is related to the dynamical structure factor $S(\mathbf{q}, \omega)$ of the scattering electron system $b^{21}$

$$
d^{2} \sigma / d \omega_{2} d \Omega=(d \sigma / d \Omega)_{\mathrm{Th}} S(\mathbf{q}, \omega),
$$

where $(d \sigma / d \Omega)_{\mathrm{Th}}$ is the well-known Thomson scattering cross section, and

$$
\hbar \omega \equiv \hbar\left(\omega_{1}-\omega_{2}\right)
$$

is the transferred energy.
Under the conditions of the impulse approximation ${ }^{2}$ (the transferred energy $\hbar \omega$ must be large compared with characteristic energies of the scattering system, and $q^{-1}$ must be small compared with the interparticle distance), the doubledifferential scattering cross section is connected with the electron momentum density $\rho(\mathbf{p})$ of the scattering system, where this relation in the nonrelativistic limit reads

$$
\begin{aligned}
d^{2} \sigma / d \omega_{2} d \Omega= & (d \sigma / d \Omega)_{\mathrm{Th}}(2 \pi)^{-3} \int d \mathbf{p} \rho(\mathbf{p}) \delta\left[\left(\hbar^{2} q^{2} / 2 m\right)\right. \\
& -(\hbar \mathbf{q} \cdot \mathbf{p} / m)-\hbar \omega] .
\end{aligned}
$$

The integral on the right-hand side of Eq. (4) contains the projection of the momentum density $\rho(\mathbf{p})$ on the scattering vector $\mathbf{q}$. If $\mathbf{q}$ points in the $z$ direction of momentum space, one can perform the integration in Eq. (4) ending up with

$$
\begin{aligned}
\int & \left.d \mathbf{p} \rho(\mathbf{p}) \delta\left[\left(\hbar^{2} q^{2} / 2 m\right)-(\hbar \mathbf{q} \cdot \mathbf{p} / m)-\hbar \omega\right)\right] \\
= & (m / \hbar q) \iint \rho(\mathbf{p}) d p_{x} d p_{y}=(m / \hbar q) J\left(p_{z}\right),
\end{aligned}
$$

where, by using Eq. (1),

$$
\begin{aligned}
p_{z}= & \mathbf{q} \cdot \mathbf{p} / q=(\hbar q / 2)-(\omega m / q)=-m c\left\{\left[\hbar \omega_{1}-\hbar \omega_{2}\right.\right. \\
& \left.-\left(\hbar^{2} \omega_{1} \omega_{2} / m c^{2}\right)(1-\cos \theta)\right] /\left[\hbar^{2} \omega_{1}{ }^{2}+\hbar^{2} \omega_{2}{ }^{2}\right. \\
& \left.\left.-2 \hbar^{2} \omega_{1} \omega_{2} \cos \theta\right]^{1 / 2}\right\} .
\end{aligned}
$$

We call $J\left(p_{z}\right)$ the Compton profile. It has been shown by Riberfors ${ }^{22}$ that, even in the relativistic case, one can factorize the DDSCS into $p_{z^{-}}, \omega_{1^{-}}$, and $\omega_{2}$-dependent relativistic cross sections (see Ref. 22 for the explicit expression), which takes the place of the Thomson cross section in Eq. (4), multiplied by the Compton profile. How experimental results can be influenced, when going beyond the impulse approximation, has been explicitly discussed in Ref. 23. As shown by Holt and Ribberfors, ${ }^{23}$ corrections to the impulse approximation, first order with respect to $1 / q$, lead to asymmetries in the Compton profile with respect to $p_{z}$, when these are extracted from the DDSCS according to Eqs. (4)-(6). Issolah et $a .^{23}$ gave expressions for the deviation of the maximum values of these Compton profiles compared with those calculated on the basis of the impulse approximation. In what follows, we will use expressions, given in Ref. 23, in order to estimate the influence of these deviations from the impulse approximation on our results.

The momentum density $\rho(\mathbf{p})$ of an inhomogeneous system can be expressed in terms of electron field operators $\Psi(\mathbf{r}, t)$,

$$
\begin{aligned}
\rho(\mathbf{p})= & (2 \pi)^{-3} \int d \mathbf{r} \int d \mathbf{r}^{\prime} \exp \left[i \mathbf{p} \cdot\left(\mathbf{r}-\mathbf{r}^{\prime}\right)\right] \\
& \times\left\langle\Psi^{+}(\mathbf{r}, 0) \Psi\left(\mathbf{r}^{\prime}, 0\right)\right\rangle,
\end{aligned}
$$

where \langle\rangle means the thermal average for the system of $N$ electrons in the volume $V$ of the crystal. We expand the field operators in Bloch waves,

$$
\Psi(\mathbf{r}, t)=\sum_{\mathbf{k}} \sum_{\nu} a_{\mathbf{k}, \nu}(t) \phi_{\mathbf{k}, \nu}(\mathbf{r}) .
$$


$a_{\mathbf{k}, \nu}$ annihilates an electron with wave vector $\mathbf{k}$ in the band $\nu$.

The Bloch wave function $\phi_{\mathbf{k}, \nu}(r)$ can be expanded in plane waves according to

$$
\phi_{\mathbf{k}, \nu}(\mathbf{r})=V^{-1 / 2} \sum_{\mathbf{G}} \alpha_{\nu}(\mathbf{k}+\mathbf{G}) \exp [i(\mathbf{k}+\mathbf{G}) \cdot \mathbf{r}]
$$

$\mathbf{G}$ is a reciprocal-lattice vector. The electron momentum density can then be written as follows:

$$
\begin{aligned}
& \rho(\mathbf{p})=\sum_{\nu, \nu^{\prime}} \sum_{\mathbf{k}} \sum_{\mathbf{G}} n_{\nu \nu^{\prime}}(\mathbf{k}) \alpha_{\nu}^{*}(\mathbf{k}+\mathbf{G}) \alpha_{\nu^{\prime}}(\mathbf{k}+\mathbf{G}) \\
& \quad \times \delta(\mathbf{k}+\mathbf{G}-\mathbf{p} / \hbar) \\
& n_{\nu \nu^{\prime}}(\mathbf{k}) \equiv\left\langle a_{\mathbf{k}, \nu}^{+}(0) a_{\mathbf{k}, \nu^{\prime}}(0)\right\rangle
\end{aligned}
$$

can be interpreted as the mean occupation number density of Bloch states, where the nondiagonal elements of the occupation number density are due to mixing between different bands caused by electron-electron interaction. Moreover, electron-electron interaction gives rise to deviations of $n_{\nu \nu}(\mathbf{k})$ from unity and zero, respectively. ${ }^{24}$ In what follows we will consider cases where the nondiagonal terms of the occupation number density are neglected, which possibly could be justified for alkali metals, as was shown by Lundquist and Lyden. $^{25}$

Equation (10), together with Eq. (5), tells us that the Compton profile of solid-state electrons is determined, on one hand, by the occupation number density, and, whenever one can neglect its nondiagonal elements, by the shape of the Fermi surface in the case of metals. On the other hand, the Bloch-wave functions, represented by their plane-wave expansion coefficients $a_{\nu}(\mathbf{k}+\mathbf{G})$, fix the shape of the Compton profile. According to Eq. (10), the contribution of the Gth plane-wave expansion coefficient to $\rho(\mathbf{p})$ is centered at $\mathbf{G}$ in momentum space, where the $\mathbf{G} \neq \mathbf{0}$ contributions are called higher-momentum components (HMC's). Like the Fermi surface around $\mathbf{G}=\mathbf{0}$, the Fermi surfaces around the $\mathbf{G}$ 's (socalled secondary Fermi surfaces) will also produce discontinuities of $\rho(\mathbf{p})$ in the case of metals, which should also produce discontinuities in the first derivative of the Compton profiles according to Eq. (5).

By evaluating Compton profiles obtained from singlecrystal samples for a larger number of different orientations of $\mathbf{q}$ (directional Compton profiles), one can reconstruct both $\rho(\mathbf{p})$ in 3D momentum space (see, e.g., Ref. 10) and, neglecting the nondiagonal elements of the occupation number density, the function

$$
N(\mathbf{k}) \equiv \sum_{\nu} n_{\nu \nu}(\mathbf{k})
$$

in the repeated zone scheme. ${ }^{7,12,26} \mathrm{We}$ will apply the socalled Fourier-Bessel method ${ }^{10}$ for this purpose, which utilizes the properties ${ }^{7}$ of the so-called reciprocal form factor ${ }^{8}$ $B(\mathbf{r})$, which is the Fourier transform of $\rho(\mathbf{p})$,

$$
B(\mathbf{r})=\int d p \rho(p) \exp (-i \mathbf{p} \cdot \mathbf{r}),
$$

so that, according to Eq. $(5), B(0,0, z)$ is obtained from the 1D Fourier transform of Compton profiles:

$$
B(0,0, z)=\int J\left(p_{z}\right) \exp \left(-i p_{z} z\right) d p_{z},
$$

if the $z$ axis in real space is assumed to be parallel to $\mathbf{q}$. The Fourier-Bessel method of reconstruction is based on the expansion of $B(\mathbf{r})$ into lattice harmonics $F_{l}(\theta, \phi),{ }^{27}$ via

$$
B(\mathbf{r})=\sum_{l} b_{l}(r) F_{l}(\theta, \phi)
$$

$\theta$ and $\phi$ are the angular variables of $\mathbf{r}$. For reconstructing $\rho(\mathbf{r})$ we need the coefficients $b_{l}(r)$ for as many lattice harmonics as can be extracted from experiment. Let their number be $N$. If we have measured a finite set of directional Compton profiles of dimension $M, N$ must obey $N \leqslant M$, since the $b_{l}(r)$ are obtained by solving the following system of linear equations:

$$
B\left(\mathbf{r}_{i}\right)=\sum_{l} b_{l}(r) F_{l}\left(\theta_{i}, \phi_{i}\right), \quad i=1, \ldots, M
$$

where the sum is over $N$ different values of $l$, according to the symmetry of the lattice under investigation. Because of Eq. (14), $\theta_{i}$ and $\phi_{i}$ are the angular variables of the $N$ different q's of the directional Compton profiles. If we write Eq. (16) in the form of a matrix equation

$$
\mathbf{B}=\mathbf{F b}
$$

one obtains, for $M>N$,

$$
\mathbf{b}=\left(\mathbf{F}^{t} \mathbf{F}\right)^{-1} \mathbf{F}^{t} \mathbf{B},
$$

and, for $M=N$,

$$
\mathbf{b}=\mathbf{F}^{-1} \mathbf{B} .
$$

Using this set of $b_{l}(r)$, we obtain $\rho(\mathbf{p})$ reconstructed by means of the inverse Fourier transform of $B(\mathbf{r})$ according to

$$
\begin{aligned}
\rho(\mathbf{p})= & \left(1 / 8 \pi^{3}\right) \sum_{l} \int d \mathbf{r} b_{l}(r) F_{l}\left(\theta_{\mathbf{r}}, \phi_{\mathbf{r}}\right) \exp (i \mathbf{p} \cdot \mathbf{r}) \\
= & \sum_{l} \rho_{l}(p) F_{l}\left(\theta_{\mathbf{p}}, \phi_{\mathbf{p}}\right) \\
& \rho_{l}(p)=\left(i^{l} / 2 \pi^{2}\right) \int_{0}^{\infty} b_{l}(r) j_{l}(p r) r^{2} d r
\end{aligned}
$$

where $j_{l}(p r)$ are spherical Bessel functions.

It was pointed out by Hansen ${ }^{10}$ that the quality of the reconstruction depends not only on the number of contributing directional Compton profiles but also on the way how the corresponding directions of the scattering vectors $\mathbf{q}$ are distributed in the irreducible triangle of their stereographic projections. Both high-symmetry directions and a nearly equal distribution of the remaining directions within that triangle are crucial, as one can demonstrate by means of an error propagation analysis, which is presented in Appendix A. The $3 \mathrm{D}$ reconstruction of $N(\mathbf{k}) \equiv \sum_{\nu} n_{\nu \nu}(\mathbf{k})$ rests on the fact that, as can be shown easily, ${ }^{7}$ the values of the reciprocal form factor $B(\mathbf{r})$ at a lattice translational vector $\mathbf{R}$ is given by 


$$
\begin{aligned}
B(\mathbf{R})= & \left(8 \pi^{3} / V\right) \sum_{\nu, \nu^{\prime}} \sum_{\mathbf{G}} \sum_{\mathbf{k}} n_{\nu \nu^{\prime}}(\mathbf{k}) \alpha_{\nu^{\prime}} *(\mathbf{k}+\mathbf{G}) \\
& \times \alpha_{\nu}(\mathbf{k}+\mathbf{G}) \exp (i \mathbf{k} \cdot \mathbf{R}),
\end{aligned}
$$

where the $\mathbf{k}$ summation is over the whole Brillouin zone. Neglecting the nondiagonal elements of the occupation number density and utilizing the normalization of the Bloch waves, we obtain

$$
B(\mathbf{R})=\left(8 \pi^{3} / V\right) \sum_{\mathbf{k}} N(\mathbf{k}) \exp (i \mathbf{k} \cdot \mathbf{R}) .
$$

Thus we can calculate $N(\mathbf{k})$ in the repeated zone scheme by means of the following Fourier expansion:

$$
N(\mathbf{k})=\left(V_{0} / 8 \pi^{3}\right) \sum_{\mathbf{R}} B(\mathbf{R}) \exp (-i \mathbf{k} \cdot \mathbf{R}),
$$

where $V_{0}$ is the volume of the elementary cell. This way we can 3D reconstruct $N(\mathbf{k})$ by using in Eq. (23) the reciprocal form factor $B(\mathbf{r})$, as reconstructed according to Eq. (16). This way, the reconstruction of $N(\mathbf{k})$ is on the same footing as the reconstruction of $\rho(\mathbf{p})$. The error propagation of the $N(\mathbf{k})$ reconstruction is shown in Appendix B. It should be mentioned that this $N(\mathbf{k})$-reconstruction method is analogous to the application of the so-called Lock-Crisp-West (LCW) theorem, ${ }^{28}$ often used in angular correlation measurements of 2- $\gamma$ radiation from positron annihilation (ACAR) done in order to obtain Fermi-surface information.

Even if we cannot neglect nondiagonal elements $n_{\nu \nu^{\prime}}(\mathbf{k})$ of the occupation number density, $B(\mathbf{R})$ contains very useful information, as shown in Appendix C, namely,

$$
B(\mathbf{R})=8 \pi^{3} \sum_{\mathbf{k}} \overline{\rho_{\mathbf{k}}} \exp (i \mathbf{k} \cdot \mathbf{R})
$$

where $\bar{\rho}_{\mathbf{k}}$ is the total contribution of all Bloch states, belonging to the same $\mathbf{k}$, to the spatially averaged electron density, according to

$$
\bar{\rho} \equiv(1 / V) \int \rho(\mathbf{r}) d \mathbf{r}=\sum_{\mathbf{k}} \overline{\rho_{\mathbf{k}}},
$$

so that

$$
\overline{\rho_{\mathbf{k}}}=\left(1 / 8 \pi^{3} N\right) \sum_{\mathbf{R}} B(\mathbf{R}) \exp (-i \mathbf{R} \cdot \mathbf{k}) .
$$

\section{EXPERIMENT AND DATA PROCESSING}

The Compton measurements were performed at the Compton beamline of HASYLAB (Hamburger, Synchrotronstrahlungs labor), described in detail in Ref. 16. Synchrotron X-rays from the HARWI-Wiggler ${ }^{2}$ of the DORIS storage ring are monochromatized by a Si (511) double crystal to 31 $\mathrm{keV}$, and sagittally focused to a spot of $8 \times 8 \mathrm{~mm}^{2}$ on the sample. Radiation scattered by $\theta=155^{\circ}$ is energy analyzed by means of a cylindrically bent $\mathrm{Si}(400)$ Cauchois-type crystal, so that the complete spectrum can be recorded simultaneously by a position-sensitive Ge-200-strip detector ${ }^{29}$ (strip width $200 \mu \mathrm{m}$, strip distance $40 \mu \mathrm{m}$ ), where each strip is connected with its own chain of preamplifier and amplifier.

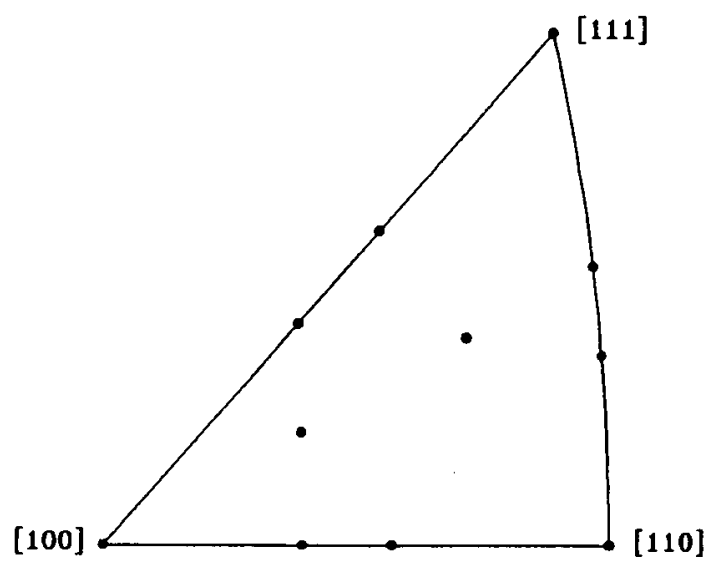

FIG. 1. Irreducible orientation triangle of cubic symmetry; measured directions of $\mathbf{q}$ are represented by points.

In order to minimize scattering from the analyzer crystal, which contributes to the background of the Compton spectra, a slit is mounted at the position of the smallest diameter of the beam trajectories between the analyzer crystal and detector.

The Li single crystal was a plate with an area of $8 \times 8 \mathrm{~mm}^{2}$ and $6 \mathrm{~mm}$ thick, mounted on a goniometer in order to bring it into different orientations with respect to q. Besides the principal directions [100], [110], and [111], another eight directions were measured, equally distributed over the irreducible orientation triangle (see Fig. 1). The Li sample, which was stored in water-free parafin oil, was cleaned from parafin by heptan, then etched by highly pure methanol to a metallic luster and again cleaned by means of xylol. After this treatment the sample was mounted immediately in the scattering chamber, which was evacuated to $10^{-5}$ mbar. After the measurement the sample had kept its metallic luster.

In each of the 11 directional Compton profiles $7 \times 10^{6}$ counts were collected. A signal-to-background ratio of 40:1 could be achieved, where the main contribution to the background is due to scattering from the analyzer crystal, from the air-path between analyzer and detector, and from the Aldetector window.

The goal of the data processing is to extract the Compton profile $J\left(p_{z}\right)$ from the measured intensity distribution $I(x)$, where $x$ is the linear position on the detector. If $U(x)$ is the background, and $M\left(p_{z}\right)$ the multiple-scattering contribution, the following relation holds:

$$
J\left(p_{z}(i)\right)+M\left(p_{z}(i)\right)=C F(E(i))[I(x(i))-U(x(i))] .
$$

$i$ stands for the channel number of the Ge-strip detector, $C$ is a normalization constant, and $F(E)$ contains the following energy-dependent corrections.

(i) The energy calibration of the detector. This is done by replacing the scattering sample by samples whose $K_{\alpha}$ and $K_{\beta}$ fluorescence lines are between the primary energy of the experiment and the maximum Compton shift recorded.

(ii) The correction with respect to the individual efficiency of the each strip of the position sensitive Ge detector, measured by illuminating the detector with $\mathrm{Ba} K_{\alpha}$ radiation from a source $1 \mathrm{~m}$ away from the detector. 
(iii) The absorption correction, where the energydependent absorption of the sample ${ }^{30}$ the Be window of the scattering chamber, the air path, and the Al-detector window were taken into account.

(iv) The scale correction $(d E / d x)^{-1}$ since one is measuring the double-differential scattering cross section $d^{2} \sigma / d \Omega d x$ and not $d^{2} \sigma / d \Omega d E$. Only the latter one is directly related to the Compton profile according to Eq. (4).

(v) The correction with respect to the energy dependence of the relativistic scattering cross section. ${ }^{22}$

(vi) The correction because of the energy dependence of the analyzer crystal reflectivity, which was approximated by means of a layer model, as proposed in Ref. 16.

The multiple-scattering contribution $M\left(p_{z}\right)$ was calculated by means of a Monte Carlo simulation written by Sakai: ${ }^{31}$ For each simulation the path of $10^{7}$ photons was followed. The relative portion of double scattering compared to single scattering was $2.7 \%$, in good agreement with calculations of Paatero and Halonen. ${ }^{32}$ The contribution of triple scattering could be neglected.

According to Eq. (23), background subtraction had to precede the energy-dependent corrections. But this procedure is only practicable if either the background is known from an independent measurement, or the background can be assumed to be linear and can thus be extrapolated using $I(x)$ values at energies larger than the primary energy on one side of the Compton profile and much smaller than the Compton shift on the other side. Since the air path between the analyzer and detector and the detector window $(3 \mathrm{~mm}$ apart from the detector surface) produce a nonlinear background, which cannot be measured independently, we have combined background subtraction and Compton profile normalization in the following iterative procedure, with the aim of obtaining empirical values of the nonlinear background on both sides of the valence Compton profile, which could then be extrapolated into the $p_{z}$ range of the valence Compton profile.

We use Eq. (27) to calculate $U(i)$ for that part of the Compton profile $\left(p_{z}<-p_{0}\right.$ or $\left.p_{z}>p_{0}\right)$, where only the core electrons are considerably contributing to the total profile,

$$
U(i)=I(i)-[C F(E(i))]^{-1}\left[J_{\text {core }}\left(p_{z}(i)\right)+M\left(p_{z}(i)\right)\right] .
$$

Here we use normalized core-electron Compton profiles, $J_{\text {core }}\left(p_{z}\right)$, as calculated for free atoms, ${ }^{33}$ assuming the influence of the solid state on $J_{\text {core }}$ to be neglegible. The normalization constant $C$ can be obtained from the normalization convention for Compton profiles,

$$
\int_{-\infty}^{+\infty} J\left(p_{z}\right) d p_{z}=Z
$$

$Z$ is the number of electrons per atom. Thus we obtain

$$
\int_{-p_{0}}^{+p_{0}} J\left(p_{z}\right) d p_{z}=Z_{\mathrm{val}}+\int_{-p_{0}}^{+p_{0}} J_{\mathrm{core}}\left(p_{z}\right) d p_{z} .
$$

From Eqs. (28) and (30), we obtain the normalization constant

$$
C=\left[Z_{\mathrm{val}}+\int_{-p_{0}}^{+p_{0}} J_{\text {core }}\left(p_{z}\right) d p_{z}+\int_{-p_{0}}^{+p_{0}} M\left(p_{z}\right) d p_{z}\right] /\left\{\int_{-p_{0}}^{+p_{0}} F(E(i))\left[I\left(p_{z}(i)\right)-U\left(p_{z}(i)\right)\right] d p_{z}\right\} .
$$

Equations (28) and (31) were considered as a system of coupled equations for $U(i)$, which were solved iteratively by least-square fitting of a fourth-order polynomial to $U(i)$ within $\left[-p_{\min },-p_{0}\right]$ and $\left[p_{0}, p_{\max }\right]$, respectively with $p_{\min }=p_{\max }=7$ a.u. and $p_{0}=2$ a.u. The iterative process was stopped, when self-consistency with respect of the constant $C$ with $\Delta C / C \leqslant 10^{-3}$ was achieved. Since the scattering angle $\theta$ of the experimental setup was not exactly known, we let $\theta$ be a free parameter. After having completed the above iterative process, using $\theta=155^{\circ}$, we determined the peak position of the total Compton profile on the energy scale. From this peak position we calculated another value of the scattering angle $\theta$, by using the relativistic counterpart ${ }^{22}$ of Eq. (6). This value was used in a second round of the above iterative process, and we proceeded until the shift of the Compton peak position became smaller than one one-thousandth of the momentum space resolution of the experiment.

We estimated the influence of deviations from the impulse approximation, as discussed in Ref. 23, on the evaluation procedure given above: According to relations of Holt and Ribberfors, ${ }^{23}$ the asymmetry of the Li $1 s$ core Compton profile, defined as $\left[J\left(p_{z}\right)-J\left(-p_{z}\right)\right] / J(0)$, has, under our experimental conditions, a maximum value of $0.9 \%$ around $p_{z}=1$ a.u., and can be completely neglected for $p_{z}>2$ a.u. Therefore, the fitting of core profiles in the range 7 a.u. $<\left|p_{z}\right|<2$ a.u. is not influenced by the asymmetry. However, we defined the zero point of the $p_{z}$ scale as coinciding with the peak position of the total profile. This is correct only for symmetric profiles. Hence an asymmetric core profile can, in principle, lead to an error in fixing of the $p_{z}$ scale. Because of the rather small asymmetry of the $1 s$ core Compton profile, this error turned out to be only \pm 0.003 a.u., and could be neglected. Taking into account the smallness of this error, the fictitious asymmetry of our valence Compton profile, which is produced, since we have subtracted a symmetric core profile from a total profile, which is, in reality, asymmetric, will not influence further physical deduction, since they all are based on averaging the $p_{z}<0$ and the $p_{z}>0$ contributions to the valence Compton profile. Using the relations of Issolah et al., ${ }^{23}$ we estimated the difference of the Li $1 s$ Compton profile maximum between the impulse approximation (hydrogenlike wave functions are used only to describe the initial state), and calculations, where hydrogenlike continuum wave functions are additionally utilized to account for the final state. Under our experimental conditions, this difference was calculated to be only $0.2 \%$, so that it could be neglected. 


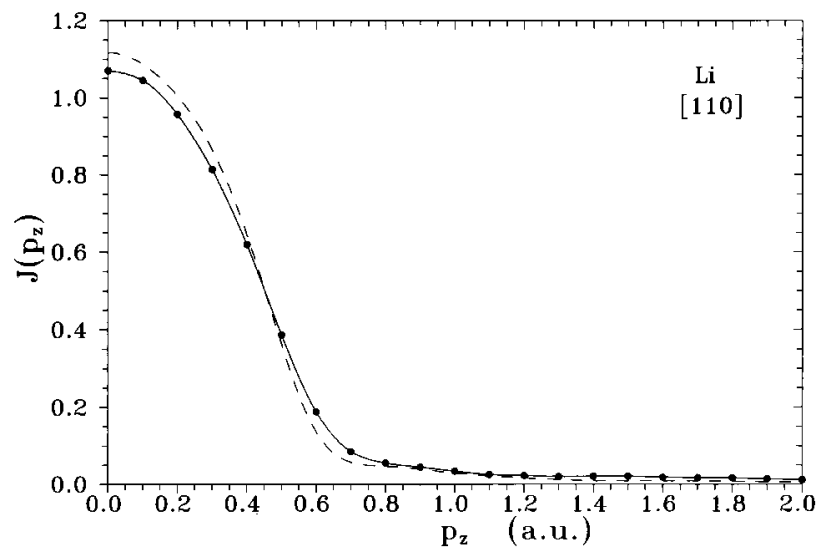

FIG. 2. Total valence-electron Compton profiles of $\mathrm{Li}$ for $\mathbf{q} \|[110]$. Experiment: Points connected by a solid line: LDA calculated. Correlation corrected (Ref. 20): dashed line.

The total momentum-space resolution of the experiment was calculated to $\Delta p_{z}=0.14$ a.u. by using a relation as given in Ref. 16, where the total energy resolution of the spectrometer is determined mainly by the lateral resolution of the position-sensitive 200-strip Ge detector $(54 \mathrm{eV})$ and by the energy resolution of the monochromator $(27 \mathrm{eV})$, whereas the reflection width of the analyzer and the influence of the analyzer bending could be neglected. This calculated energy resolution is, within the experimental error, in agreement with the full width at half maximum (FWHM) of the elastically scattered line. The finite angular width of the incident and of the scattered radiation is of negligible influence. All theoretical Compton profiles shown in what follows are convoluted with a Gaussian whose FWHM is equal to 0.14 a.u.

\section{RESULTS}

\section{A. Total Compton profiles, difference profiles}

Figure 2 shows the Li valence-electron Compton profile of $\mathrm{Li}$ after subtraction of the core contribution for $\mathbf{q} \|[110]$, where the data points are the result both of averaging the $p_{z}<0$ and $p_{z}>0$ contributions and of interpolating on the $p_{z}$ grid of the experiment. The relative error at $p_{z}=0$ is $0.27 \%$, and cannot be represented in form of an error bar. Together with the experimental data, LDA theoretical calculations ${ }^{18}$ are plotted, which were correlation corrected ${ }^{20}$ and convoluted with the experimental resolution. The discrepancy between calculations and experiment is far from the experimental error, in spite of the fact that in the higher momentum region, which represents the contribution of the core othogonalization to the valence-electron Compton profile, the agreement is quite satisfactory. In that region the experimental results of Ref. 18 disagree systematically with the LDA calculations, possibly due to an incomplete allowance for a nonlinear background. As a consequence, the overall discrepancy between calculation and experiment is also larger in Ref. 18 than in our results. In Sec. V we provide an explanation for the remaining discrepancy in our experiment, referring to a many-particle treatment of the Comptonscattering process.

In Fig. 3, the difference between the experimental total directional Compton profiles and the corresponding LDA

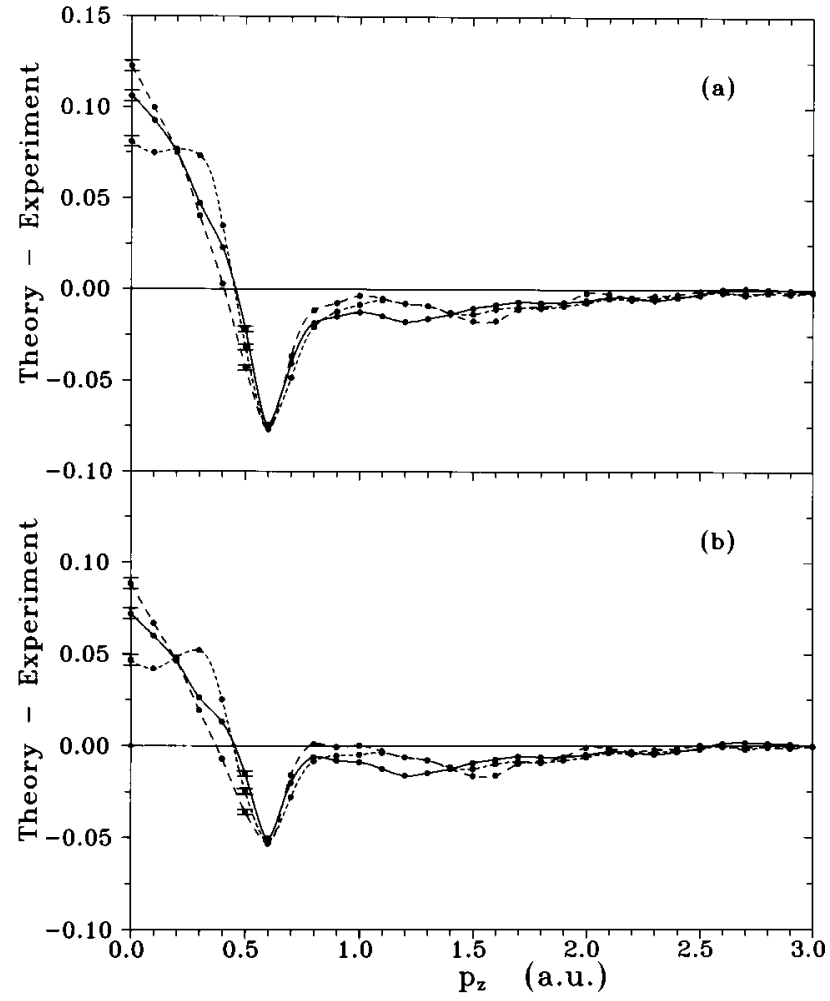

FIG. 3. Difference between LDA-calculated and measured valence-electron Compton profiles: (-----) q\| [110]; (-) q\| [100]; (---) q\| [111]. (a) LDA without correlation correction. (b) LDA with correlation correction according to Ref. 20.

calculations [(a) without and (b) including correlation correction according to Ref. 20] are plotted for different $\mathbf{q}$ directions. An orientation dependence outside the experimental error is evident, and seems to be due to the fact that the theory generally overestimates the contributions of the secondary Fermi surfaces [see Sec. II, Eq. (10)] to the directional profiles. This is demonstrated in Fig. 4, where, within each secondary Fermi surface of the type 110, regions are hatched which contribute strongly to the momentum density, due to a large strength of the relevant umklapp processes: The hatched regions of altogether six secondary Fermi surfaces contribute to the [111] directional Compton profile at $p_{z}=0$ (section $A A^{\prime}$ through the momentum space, perpendicular to the [111]-direction in Fig. 4), whereas only two contribute to the [110]-profile at $p_{z}=0$, so that the difference between theory and experiment at $p_{z}=0$ in Fig. 3 is maximum for the [111] directional profile and minimum for the [110] directional profile. Around $p_{z}=0.4$ a.u. the relations invert. Now the hatched regions of four secondary Fermi surfaces contribute to the [110] profile (dashed section $D D^{\prime}$ in Fig. 4), whereas no hatched region is involved the [111] profile (dashed section $B B^{\prime}$ ), so that the difference between theory and experiment at $p_{z}=0.4$ a.u. is maximum for the [110] profile and minimum for the [111] profile. In Sec. V we propose a scheme which may qualitatively explain the reason for this overestimation of the contributions of the secondary Fermi surfaces in the theoretical calculation. As a consequence of this general phenomenon the directional differences, as shown in Fig. 5, also have larger amplitudes in the LDA calculations than in the experiment, whereas the 


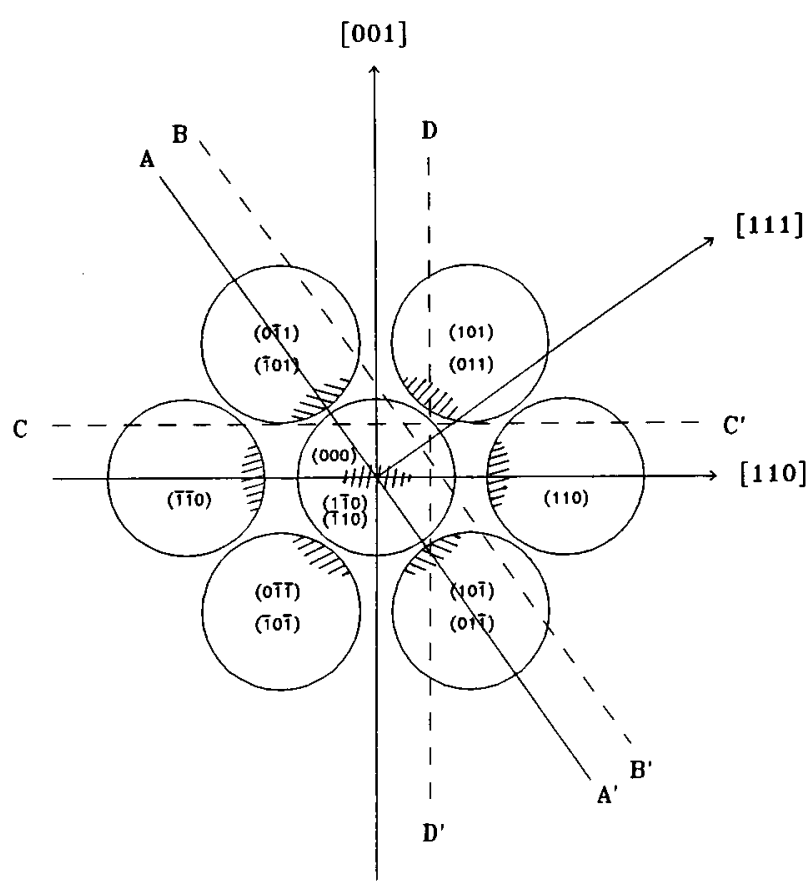

FIG. 4. Projection of the primary and the 12 110-type secondary Fermi spheres of Li on the (110) plane. The hatched regions of the secondary Fermi spheres are strongly occupied due to umklapp processes at the 110-type Brillouin-zone boundaries. The solid lines are traces of the planes of integration in $\mathbf{p}$ space corresponding to $p_{z}=0$ : the line $A A^{\prime}$ for $\mathbf{q} \|[111]$, the line parallel to the [110] axis for $\mathbf{q} \|[001]$; and the line parallel to the [001] axis for $\mathbf{q} \|[110]$. The dashed lines are traces of the planes of integration in $\mathbf{p}$ space corresponding to $p_{z}=0.4$ a.u.: $B B^{\prime}$ for $\mathbf{q} \|[111], C C^{\prime}$ for $\mathbf{q} \|[001]$, and $D D^{\prime}$ for $\mathbf{q} \|[110]$.

experimental zero passages and peak positions agree quite well with the calculations, thus indicating a proportional reduction of the secondary Fermi surfaces' contribution.

\section{B. 3D reconstruction of the momentum density}

By using 11 directional Compton profiles, we reconstructed the 3D momentum density $\rho(\mathbf{p})$ according to Eqs. (13)-(20), where the profiles were interpolated on an equidistant $p_{z}$ scale $\left(\Delta p_{z}=0.025\right.$ a.u. $)$, and $B(z)$ values up to $z_{\max }=30$ a.u. were taken into account. The limiting value $z_{\max }$ was chosen such that $\left|B\left(z_{\max }\right)\right| \cong \sigma_{B}$, where $\sigma_{B}$ is the standard deviation of $B(z)$. In order to call special attention to the physically interesting anisotropy of the momentum density, in Fig. 6(a) we plot the anisotropic part $\Delta \rho(\mathbf{p})$ of $\rho(\mathbf{p})$ in the $\Gamma N P H$ plane in the form of a level diagram by subtracting the $l=0$ contribution in Eq. (20). Additionally, the corresponding error map of $\Delta \rho(\mathbf{p})$ is plotted in Fig. 6(b), calculated according to relations given in Appendix A. One can easily verify that the only structures of $\Delta \rho(\mathbf{p})$, which are clearly beyond experimental error, are the double peak around the (110) Brillouin-zone boundary near the $N$ point, and the depression in the $\Gamma H$ direction near $p_{F}$ (0.58 a.u.). The strong oscillatory behavior of $\Delta \rho(\mathbf{p})$ between 0 and 0.4 a.u. along $\Gamma H$ seems to be an artefact of the reconstruction procedure, since the oscillation amplitudes are of the same order as the error in this region of momentum space. The

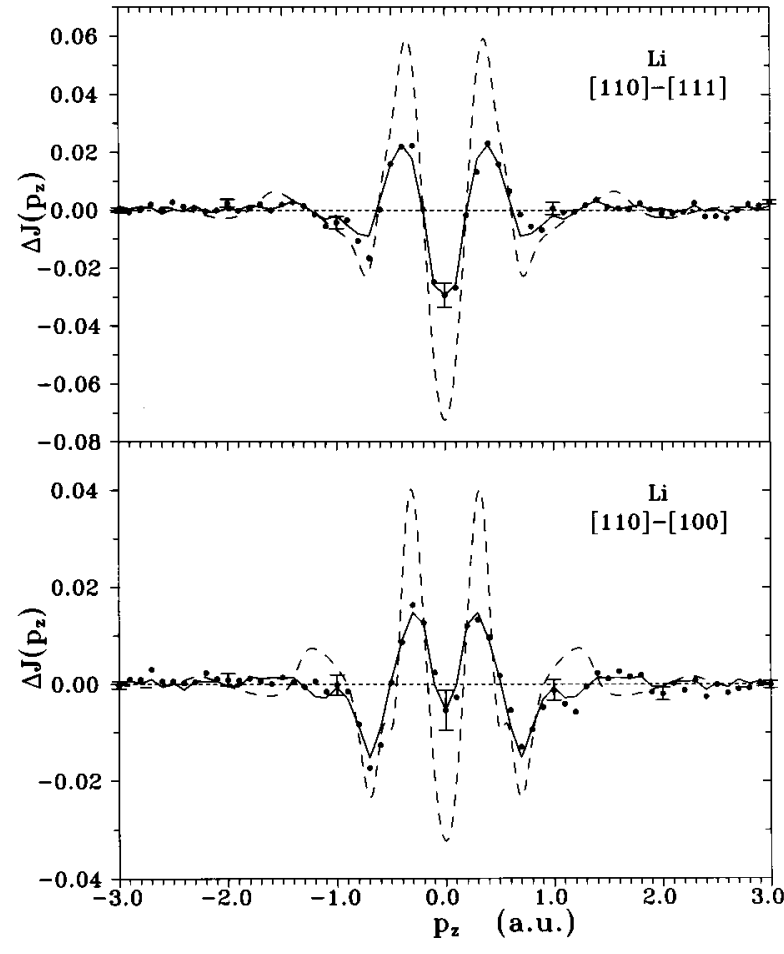

FIG. 5. Directional Compton profile differences of Li. Upper panel: [110]-[111]. Lower panel: [110]-[100]. Points: experimental data. Solid line: experimental data obtained by averaging the $p_{z}<0$ and $p_{z}>0$ parts of the Compton profiles. Dashed line: LDAcalculated directional differences of Li Compton profiles.

same applies to the peak midway $\Gamma P$. The peak of $\Delta \rho(\mathbf{p})$ near $N$ within the first Brillouin zone can be attributed to the bulge of the Fermi surface in the [110] direction, which will be analyzed in more detail in connection with the reconstruction of the occupation number density in Sec. IV C. The depression in the $\Gamma H$ direction near $p_{F}$ can be attributed to a flattening of the free-electron Fermi sphere in the [100] direction (see Sec. IV C). The second peak near $N$, outside the first Brillouin zone, is a clear indication of a highermomentum component due to the secondary Fermi surface centered at $\mathbf{G}_{110}$. As shown in Fig. 6(c); the double-peak structure of $\Delta \rho(\mathbf{p})$ near the $N$ point as well as the depression in the $\Gamma H$ direction near $p_{F}$ are also found, when the $3 \mathrm{D}$ reconstruction procedure is applied to 11 LDA-calculated directional Compton profiles (Ref. 18) (convoluted with the experimental resolution) with the same $q$ orientations as in the experiment. Traces of these (110) higher-momentum components were also found in 2D ACAR measurements of Oberli et al. ${ }^{34}$

\section{3D reconstruction of the occupation number density and of the Fermi surface}

We also performed a $3 \mathrm{D}$ reconstruction of the occupation number density $N(\mathbf{k})$ in the repeated zone scheme according to Eq. (23) by using the interpolated values of $B(\mathbf{r})$ as obtained in the course of the $\rho(\mathbf{p})$ reconstruction. The result is plotted in Fig. 7(a) for the $\Gamma N P H$ plane in the form of a level diagram. In Fig. 7(b), the error map of the 3D reconstruction of the occupation number density is plotted, as obtained by means of relations given in Appendix B. 

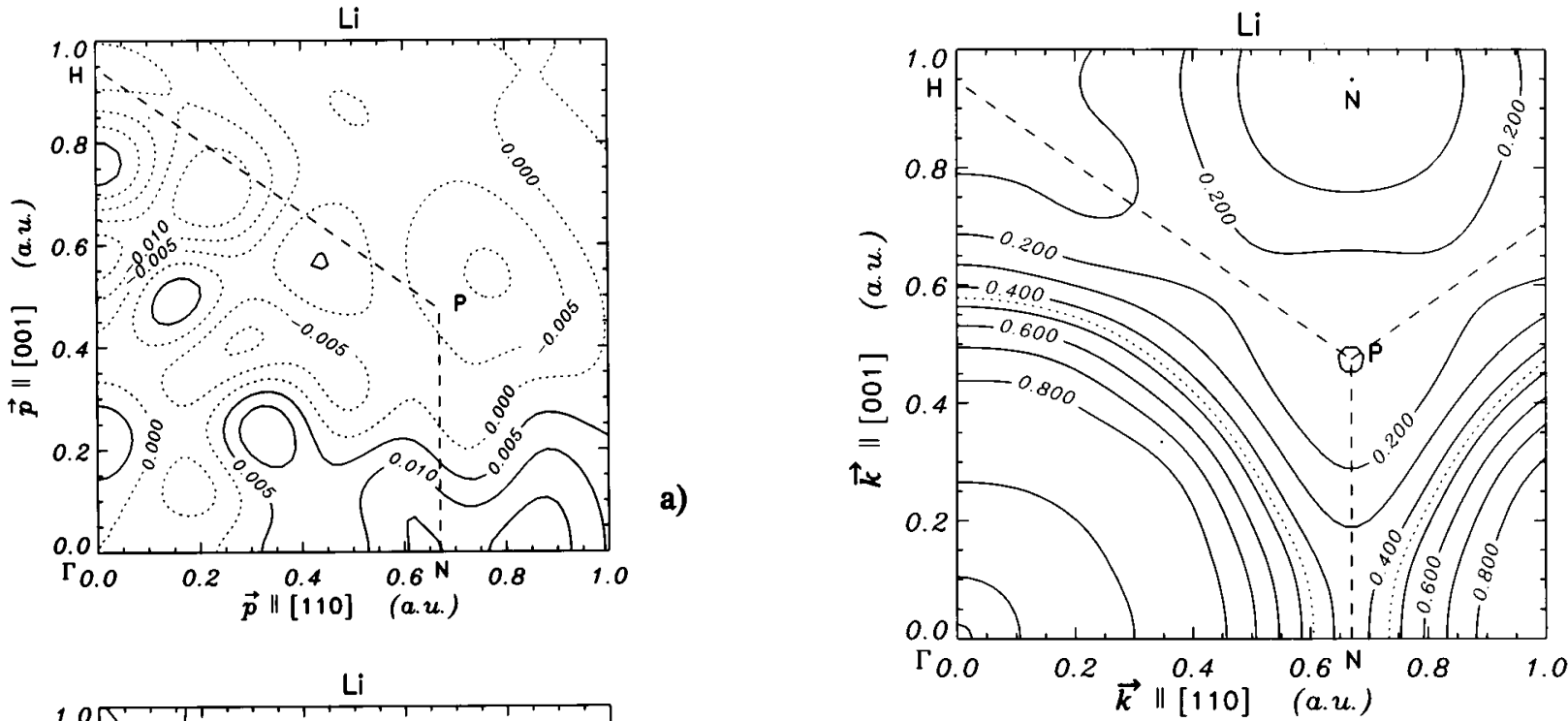

a)

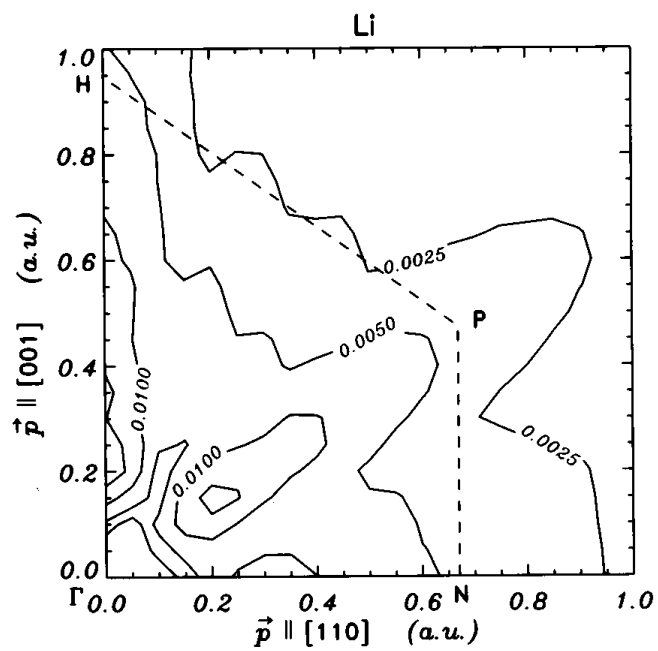

b)
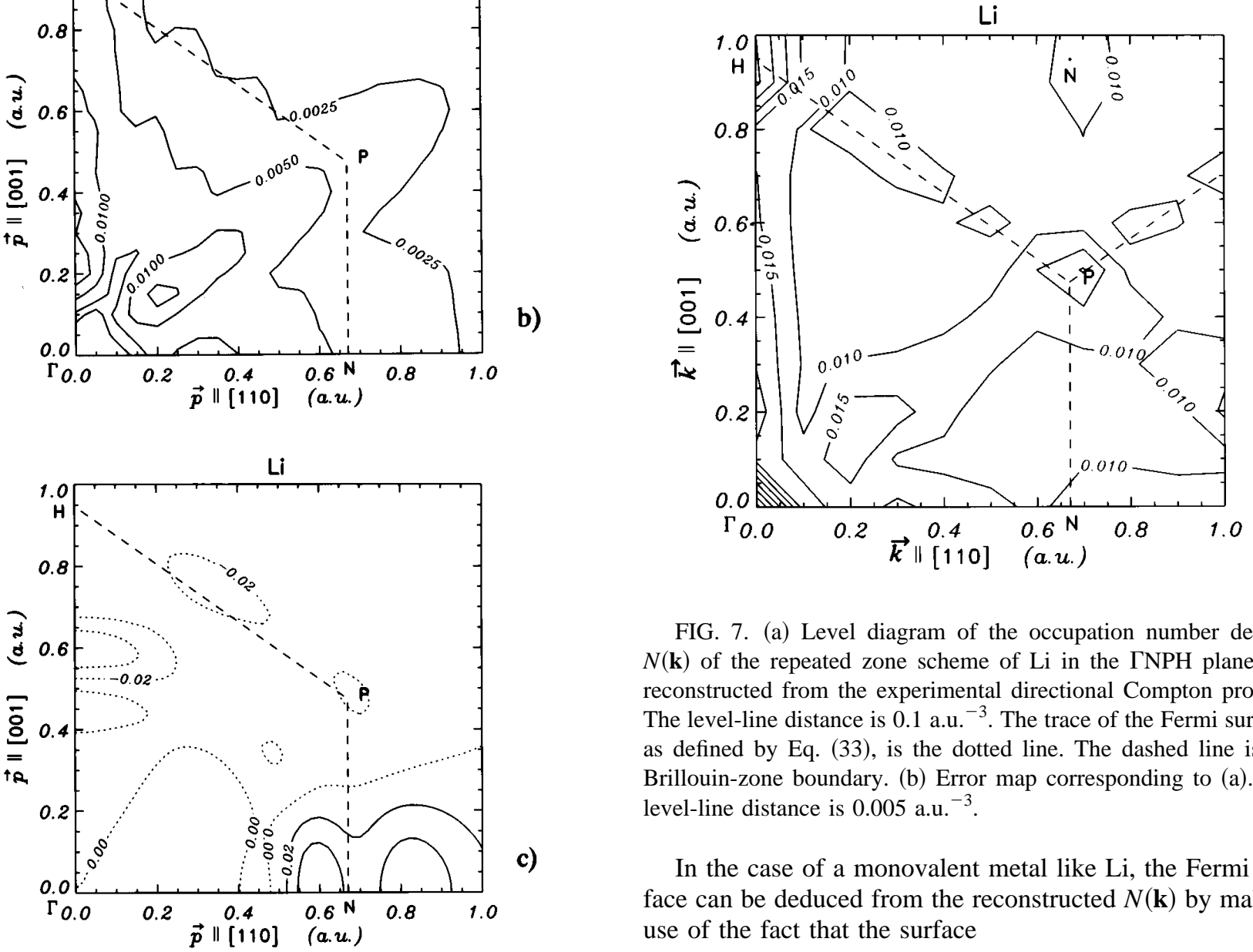

b)

FIG. 7. (a) Level diagram of the occupation number density $N(\mathbf{k})$ of the repeated zone scheme of $\mathrm{Li}$ in the $\Gamma \mathrm{NPH}$ plane, 3D reconstructed from the experimental directional Compton profiles. The level-line distance is 0.1 a.u. $^{-3}$. The trace of the Fermi surface, as defined by Eq. (33), is the dotted line. The dashed line is the Brillouin-zone boundary. (b) Error map corresponding to (a). The level-line distance is 0.005 a.u. $^{-3}$.

In the case of a monovalent metal like $\mathrm{Li}$, the Fermi surface can be deduced from the reconstructed $N(\mathbf{k})$ by making use of the fact that the surface

$$
N(\mathbf{k})=\text { const }=c,
$$

momentum density in the $(1 \overline{1} 0)$ plane, $3 \mathrm{D}$ reconstructed from the experimental directional Compton profiles. Solid lines: positive values of $\Delta \rho(\mathbf{p})$; dotted lines: negative values of $\Delta \rho(\mathbf{p})$; level-line distance: 0.005 a.u. $^{-3}$; dashed line: Brillouin-zone boundary. (b) Error map corresponding to (a); the level-line distance as in (a). (c) Level diagram of the anisotropic part, $\Delta \rho(\mathbf{p})$, of the Li momentum density in the $(1 \overline{10})$ plane, $3 \mathrm{D}$ reconstructed from the LDA-calculated directional Compton profiles; level-line distance: 0.02 a.u. $^{-3}$; all else, see the legend (a).

which defines the Fermi surface, has to enclose the volume of the corresponding free-electron Fermi sphere with radius $k_{F 0}$ :

$$
\int_{N(\mathbf{k}) \geqslant c} d \mathbf{k}=\frac{4}{3} \pi k_{F 0}^{3},
$$

where 


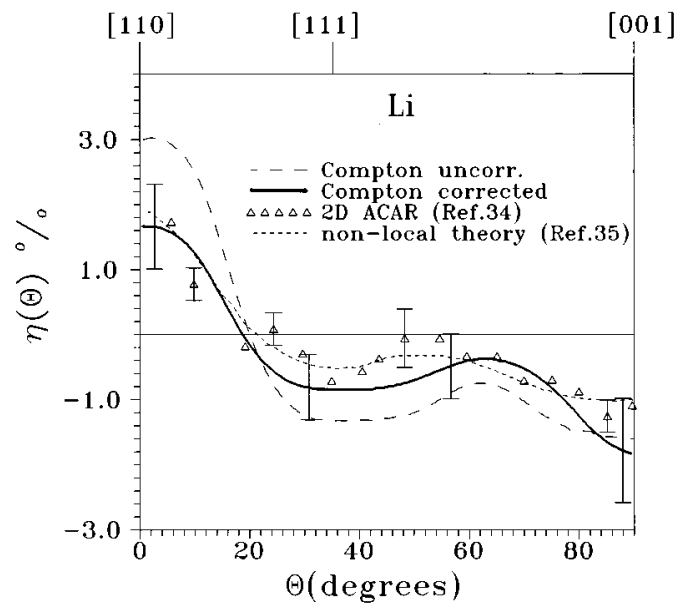

FIG. 8. Anisotropy of the Li Fermi surface as defined by Eq. (35). Long-dashed line: uncorrected data taken from the 3D reconstruction of the occupation number density using the experimental directional Compton profiles. Solid line: data from the 3D reconstruction, but corrected with respect to a systematic error (see text). Open triangles: 2D ACAR measurements of Oberli et al. (Ref. 34); short-dashed line: nonlocal calculations of MacDonald (Ref. 35).

$$
k_{F 0}=\left(3 \pi^{2} n_{e}\right)^{1 / 3},
$$

and $n_{e}$ is the valence-electron density.

The trace of the Fermi surface in the $\Gamma N P H$ plane, determined this way, is marked in Fig. 7(a) by a dotted line. This way the Fermi radius can be determined for arbitrary directions in Bloch wave-vector space. We characterize the Fermi-surface anisotropy by the following parameter $\eta(\hat{\mathbf{k}})$ :

$$
\eta(\hat{\mathbf{k}})=100\left[\left(k_{F}(\hat{\mathbf{k}})-k_{F 0}\right) / k_{F 0}\right],
$$

which is plotted in Fig. 8 as a function of the angle $\Theta$ between $\hat{\mathbf{k}}$ and the [110] direction in the (110) plane. The longdashed curve is directly deduced from Eqs. (32)-(34) using the reconstructed occupation number density. But this result still contains a systematic error, since the $3 \mathrm{D}$ reconstruction procedure of the occupation number density produces an artificial additional anisotropy of $N(\mathbf{k})$, which can be estimated the following way: We performed the $3 \mathrm{D}$ reconstruction procedure by using, instead of the 11 measured directional Compton profiles, 11 identical profiles, namely, the orientation averages of the directional profiles. In spite of starting with an isotropic distribution one ends up with a certain anisotropy of $N(\mathbf{k})$, due to the fact that we have pressed the anisotropic crystal symmetry of the bcc lattice upon the isotropic momentum-space distribution. Since the whole reconstruction procedure consists of linear operations, this artificial anisotropy can be subtracted from the long-dashed curve in Fig. 8, ending up with the solid curve of Fig. 8, which represents the Li Fermi-surface anisotropy. The error bars in Fig. 8 are based on the error map of $N(\mathbf{k})$, as shown in Fig. 7(b). In Fig. 8, we have also included the 2D ACAR results of Oberli et al. ${ }^{34}$ and the calculations of McDonald, ${ }^{35}$ which used nonlocal exchange and correlation terms in their massoperator formalism. Both are in excellent agreement with our measurements. But it must be stressed that our result has been directly derived from the reconstructed occupation number density distribution and, that we have not used any parametrized model of the momentum distribution or the Fermi surface in order to obtain the shape of the latter, contrary to the procedure for obtaining the $2 \mathrm{D}$ ACAR results, where the Fermi radii were the result of comparing the first derivative of the 2D ACAR curves, with corresponding curves deduced from a parametrized model of the Li momentum density. Moreover, these procedures are based on the so-called LCW theorem, ${ }^{28}$ which has only a very limited applicability for positron annihilation due to the influence of the positron wave function, which is not taken into account in the LCW theorem.

The comparison of our experimental Fermi-surface anisotropy with additional experiments and calculations of other authors is presented in Table I, where we used the parameter $\delta$, defined by

$$
\delta=100\left[\left(k_{F}(110)-k_{F}(001)\right) / k_{F 0}\right],
$$

\begin{tabular}{|c|c|c|}
\hline Reference & Method & $\delta(\%)$ \\
\hline This work & Compton scattering & $3.6 \pm 1.1$ \\
\hline Oberli et al. (Ref. 34) & 2D ACAR & $2.8 \pm 0.6$ \\
\hline Hunt, Reinders, and Springford (Ref. 36) & dHvA & $4.8 \pm 0.3$ \\
\hline Randles and Springford (Ref. 37) & $\mathrm{dHvA}$ & $2.6 \pm 0.9$ \\
\hline Sakurai et al. (Ref. 18) & Compton scattering & $4.1 \pm 1.0$ \\
\hline Rajput et al. (Ref. 40) & 2D ACAR & $4.7 \pm 0.2$ \\
\hline Donaghy and Stewart (Ref. 38) & 1D ACAR (long slit) & 5.0 \\
\hline Paciga and Williams (Ref. 39) & 2D ACAR (rotating specimen) & 2.9 \\
\hline \multirow[t]{2}{*}{ Rasolt, Nickerson, and Vosko (Ref. 41) } & $\begin{array}{l}\text { orthogonalized plane wave (OPW) LDA (local) } \\
\text { theory }\end{array}$ & 5.5 \\
\hline & OPW (nonlocal mass operator) & 1.4 \\
\hline \multirow[t]{2}{*}{ MacDonald (Ref. 35) } & $\begin{array}{l}\text { linear augmented plane-wave (LAPW) LDA (local) } \\
\text { theory }\end{array}$ & 6.0 \\
\hline & LAPW (nonlocal mass operator) & 3.7 \\
\hline Sakurai et al. (Ref. 18) & Korringa-Kohn-Rostoker (KKR) LDA (local) theory & 5.6 \\
\hline Rajput et al. (Ref. 40) & KKR LDA (local) theory & 5.6 \\
\hline
\end{tabular}

TABLE I. Maximum Fermi-surface anisotropy of Li in experiment and theory. 
in order to describe the maximum deviation of the Li Fermi surface from sphericity. Our results on the asphericity of the Li Fermi surface agree, within the experimental errors, with other Compton measurements, ${ }^{18}$ with the $2 \mathrm{D}$ ACAR results of Oberli et al., ${ }^{34}$ and also with the de Haas-van Alphen measurements, ${ }^{36,37}$ where the latter were performed in a polycrystalline state on small clusters in order to supress the Martensitic transition, so that the Fermi-surface anisotropy could not be obtained directly. The early long-slit (1D) ACAR measurement ${ }^{38}$ does not contain error bars, and may suffer from the double integration in momentum space. Moreover, the evaluation of this experiment did not include corrections for the anisotropy of the higher-momentum components. Those corrections were performed in the rotatingspecimen ACAR experiment of Paciga and Williams ${ }^{39}$ which reduced the Fermi-surface anisotropy by a factor of 1.6. Only the 2D ACAR results of Rajput et al. ${ }^{40}$ seem to deviate significantly from all the other experimental results. Nevertheless, details of the procedure used by the latter authors to obtain the Fermi-surface asphericity are missing, so that it is difficult to come to a final judgment. Concerning the calculations of the Li Fermi-surface anisotropy, one can find a general trend: When compared with the average experimental results, all calculations using a local approach, when handling exchange and correlation within a density-functional scheme, seem to overestimate the Fermi-surface asphericity, whereas the nonlocal approaches fit the experiment much better. This fact was first stated by Rasolt, Nickerson, and Vosko, ${ }^{41}$ and were confirmed by MacDonald. ${ }^{35}$ Therefore, the rather high values of $\delta$, found in the most recent calculations of Raijput et $a l .{ }^{40}$ and Sakurai et al. ${ }^{18}$ may also suffer from local treatment of exchange and correlation.

The direct access to the occupation number density $N(\mathbf{k})$ by the $3 \mathrm{D}$ reconstruction method applied to directional Compton profiles offers a unique possibility to test the theoretical treatment of exchange and correlation in calculations of $N(\mathbf{k})$. Those calculations have so far been done only for homogeneous systems, by Daniel and Vosko $^{24}$ within the limits of the random-phase approximation (RPA), by $\mathrm{Lam}^{42}$ including some of the exchange terms, by Overhauser ${ }^{43}$ using his plasmon-pole model together with a local-field correction of the static dielectric function, by Lantto ${ }^{44}$ making a Fermi hypernetted-chain calculation, and by Takada and Yasuhara $^{45}$ applying an effective potential expansion together with a self-consistency relation between $N(k)$ and the correlation energy per electron $e_{c}$ known very accurately from Monte Carlo calculations. ${ }^{46}$ All these calculations have held in common a value $N(0)<1$, a discontinuity $z<1(z$ is the renormalization factor) at the Fermi momentum $k_{F}$ and a tail for $k>k_{F}$ which behaves like $\left(k / k_{F}\right)^{-8}$. But the parameters which characterize these common features took very different values in the various theoretical treatments. The physical reason for the reduction of the discontinuity at the Fermi momentum and for the appearance of a tail for $k>k_{F}$ was clarified by Lundquist: ${ }^{47}$ The spectral function $A(k, \omega)$ exhibit a coupled mode of a hole and a plasmon (the socalled plasmaron) of appreciable spectral weight, whose width on the $\omega$ scale is not zero at $k=k_{F}$. The spectral weight of this mode is thus directly coupled to the value of $z$. To what extent the presence of the lattice potential may change the characteristics of $N(\mathbf{k})$ for simple metals [besides

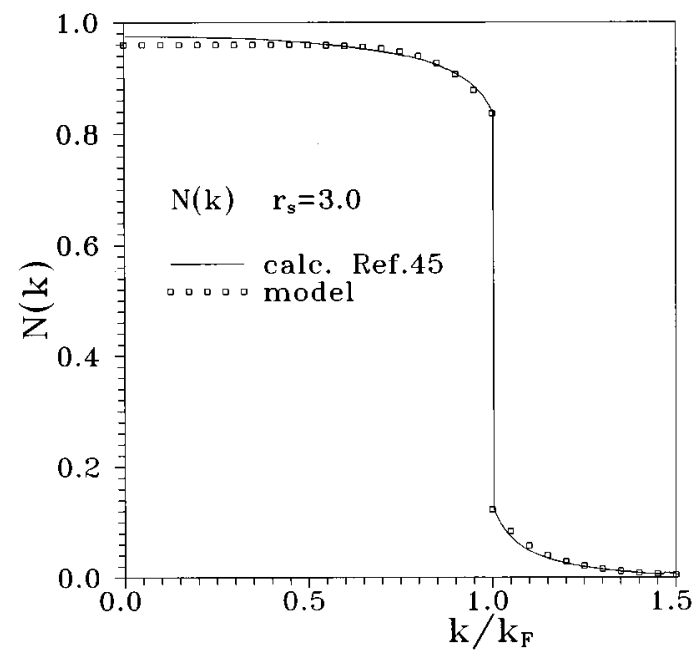

FIG. 9. Squares: model occupation number density $N(k)$ according to Eqs. (37) and (38) with $z=0.713$. Solid line: $N(k)$ calculated by Takada and Yasuhara (Ref. 45) for $r_{s}=3.0$.

the appearance of nondiagonal terms $n_{\nu \nu^{\prime}}(\mathbf{k})$, as mentioned in Sec. II] has not yet been proved by corresponding calculations of the spectral density functions. Therefore, in order to confront the occupation number densities of the various theoretical treatments with the experiment, we were left to the jellium results of $N(k)$. For that reason we constructed the following model $N(k)$, which contains $z$ as a single parameter:

$$
N(k)= \begin{cases}(1-a)-\frac{1}{2}(1-a-z)\left(k / k_{F}\right)^{8} & \text { for } k<k_{F} \\ \frac{1}{2}(1-a-z)\left(k_{F} / k\right)^{8} & \text { for } k>k_{F},\end{cases}
$$

where $a$ is determined by the normalization condition

$$
4 \pi \int_{0}^{\infty} N(k) k^{2} d k=(4 \pi / 3) k_{F}^{3}
$$

to $a=\frac{9}{64}(1-z)$. How well this model fits most recent calculations, is shown in Fig. 9: Our model is plotted against the $N(k)$ of Takada and Yasuhara ${ }^{45}$ for $r_{s}=3$, where $z=0.713$ (see Fig. 4 of Ref. 45).

By using this model $N(k)$, we calculated the corresponding model Compton profile on the sampling distance of the experiment, and convoluted it with the experimental resolution. Then we performed the $N(\mathbf{k})$ reconstruction, by using, instead of the experimental directional profiles, 11 identical model profiles, to whom we attributed the $\mathbf{q}$ directions of the experimental ones. Finally, the constant $c$ was determined according to Eq. (33). In Fig. 10 the model-reconstructed $N(\mathbf{k}) / c$ in the [100] direction for different values of the renormalization factor $z$ are compared with the experimental ones around $k_{F 0}$, with the rather surprising result that the experiment fits the model calculations best for $z=0.1 \pm 0.1$, which is by far smaller then all theoretical jellium results for $r_{s}=3.3$ obtained so far, ranging from $z=0.5$ (RPA, Ref. 24) to $z=0.75$ (Ref. 44). This result is a very strong indication of a considerable deviation of $N(\mathbf{k})$ in crystalline Li from its jellium counterpart, whereas the "experimental" value of $z$, obtained above, must not be considered that serious, since 


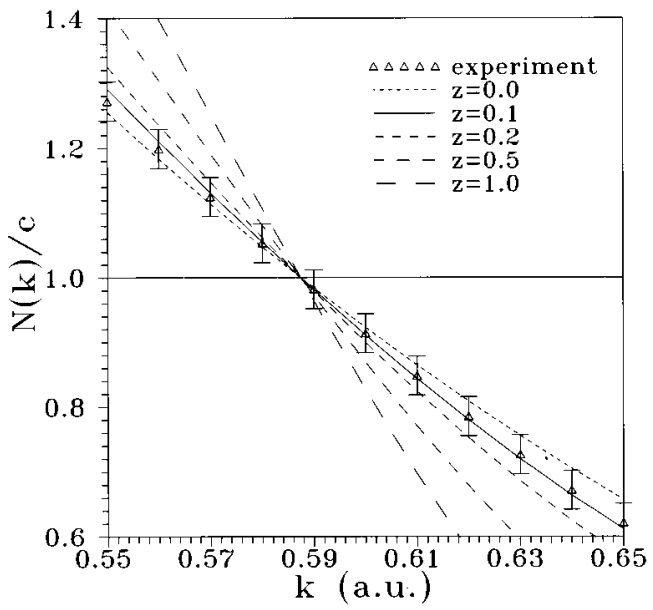

FIG. 10. The assignment of the different line styles; see the inset. Normalized occupation number density $N(k) / c$ is in the [100] direction, as reconstructed from model jellium $\left(r_{s}=3.3\right)$. Compton profiles for different values of the renormalization factor $z$. Triangles with error bars: normalized occupation number density $N(k)$ reconstructed from the experimental directional Compton profiles of $\mathrm{Li}$.

we fitted a simple one-parameter model $N(k)$ to the experiment, so that deviations from the jellium model can also be the result of a behavior of the crystal $N(\mathbf{k})$, which is different from the jellium model in other respects besides different values of $z$.

Nevertheless, one may speculate that it is the opening of additional channels for collective excitations in the crystalline solid, the so-called zone-boundary collective states ${ }^{48,49}$ observed in $\mathrm{Li}$ (Ref. 50) and Be, ${ }^{51}$ which may enhance the contribution of the above-mentioned coupled modes of holes and collective excitations, thus diminishing $z$ accordingly. On the other hand, the whole reconstruction procedure for $N(\mathbf{k})$ is based on the assumption that the nondiagonal elements of $n_{\nu \nu^{\prime}}(\mathbf{k})$ can be neglected in the case of Li, as estimated by Lundquist and Lyden. ${ }^{25}$ If this was not the case, the above comparison between model calculated and experimental values of $N(\mathbf{k})$ would lose its foundation. In that case it is not $N(\mathbf{k})$ that is plotted in Figs. 7 and 10, but, according to Eq. (26), the quantity $\overline{\rho_{\mathbf{k}}}$, the kth contribution to the spatially averaged electron density. Of course, the determination of the Fermi-surface anisotropy is also based on the neglection of the nondiagonal elements of $n_{\nu \nu^{\prime}}(\mathbf{k})$, though the contribution of these nondiagonal elements seem to be much more isotropic due to the interband mixing. So it is time to find appropriate theoretical treatments to clarify this unsatisfying situation.

\section{SELF-ENERGY EFFECTS IN THE COMPTON-SCATTERING PROCESS}

Two prominent discrepancies between our Li Compton results and corresponding LDA calculations need explanation, at least a qualitative one, that is, the lower experimental values of the total Compton profile for small $p_{z}$ compared with the calculated ones (see Fig. 2), and the smaller contributions of the secondary Fermi surfaces to the directional profiles in the experiment in comparison with the calcula- tions (see Fig. 3). In what follows, we will show that a critical investigation of self-energy effects connected with the Compton-scattering process may offer a unique explanation of these discrepancies.

For that reason we go back to Eq. (2), where the doubledifferential scattering cross section of the Compton process was connected with the dynamic structure factor $S(\mathbf{q}, \omega)$. It was shown by $\mathrm{Ng}$ and Dabrowski ${ }^{52}$ that, as far as electronhole-pair excitations dominate $\mathbf{S}(\mathbf{q}, \omega)$, self-energy effects on $S(\mathbf{q}, \omega)$, including of-shell contributions, can be obtained via the spectral density function $A(\mathbf{p}, E)$,

$$
\begin{aligned}
S(\mathbf{q}, \omega)= & (1 / \rho \pi) \int_{-\hbar \omega}^{0}(d E / 2 \pi) \int\left(d \mathbf{p} / 8 \pi^{3}\right) A(\mathbf{p}, E) A(\mathbf{p} \\
& +\mathbf{q}, E+\hbar \omega),
\end{aligned}
$$

where $\rho$ is the electron density.

In a Compton-scattering process the momentum and energy transfer are so large that the self-energy effects on $A$ (p $+\mathbf{q}, E+\hbar \omega)$ consist mainly of a finite width of the order of half the free-electron plasmon energy $E_{p}$, due to the imaginary part of the self-energy, as has been shown for jellium by Lundquist, ${ }^{53}$ within the limits of the RPA. Thus this selfenergy effect is adequately described by an additional convolution of LDA-calculated Compton profiles by a Lorentzian with a FWHM of $0.5 \times E_{p}$, which is $4 \mathrm{eV}$ in the case of Li. Since in our case the experimental energy resolution is of the order of $50 \mathrm{eV}$, this effect is of minor significance. But it may play a role, whenever a much higher-energy resolution, of the order of $1 \mathrm{eV}$, is aspired to, ${ }^{54}$ in order to obtain a corresponding high-momentum-space resolution. In these cases this self-energy effect sets natural limits to what a momentum space resolution can be achieved. The self-energy effect on the spectral density function $A(\mathbf{p}, E)$ in Eq. (39) is more important, since $A(\mathbf{p}, E)$ has a characteristic structure for $E<0$ and $p<p_{F}$, as shown for jellium by Lundquist. ${ }^{47}$ This structure consists of a quasiparticle peak, whose dispersion follows the free-particle one, and a second peak shifted by roughly $1.5 \times E_{p}$ to lower energies. The second peak is attributed to a bound hole-plasmon state, the so-called plasmaron, and contains up to $30 \%$ of the total spectral density. The double-peak structure leads to a doubling of the measured Compton profile with two components shifted by $1.5 \times E_{p}$. We have calculated the effect of the self-energy on the shape of a Compton profile within the limits of the random-phase approximation for a free-electron system of the density of $\mathrm{Li}$ under the conditions of our Li Compton experiment $\left(q=32 \times k_{F}\right)$ by using Eq. (39). In Figs. 11(a)11(c) three spectral density functions for $p=0,0.5 k_{F}$, and $0.7 k_{F}$, respectively, are shown, which are contributing to $A(\mathbf{p}, E)$ in Eq. (39). In Fig. 11(d) a representative spectral density function for $|\mathbf{p}+\mathbf{q}|=32 \times k_{F}$ is plotted, which stands for $A(\mathbf{p}+\mathbf{q}, E+\hbar \omega)$ in Eq. (39). In Fig. 12 the self-energy effect on $S(q, \omega)$, which according to Eq. (2) is proportional to the Compton profile, is presented. The contributions of the quasiparticle peak $(\sim 70 \%)$ and of the plasmaron peak $(\sim 30 \%)$, respectively, are specified. Additionally a Compton profile [(in terms of $S(q, \omega))$ ] is plotted, which is correlation corrected according to Ref. 20, and where this correction has been calculated on the same footing as the self-energy correction. The self-energy correction according to Eq. (39) 

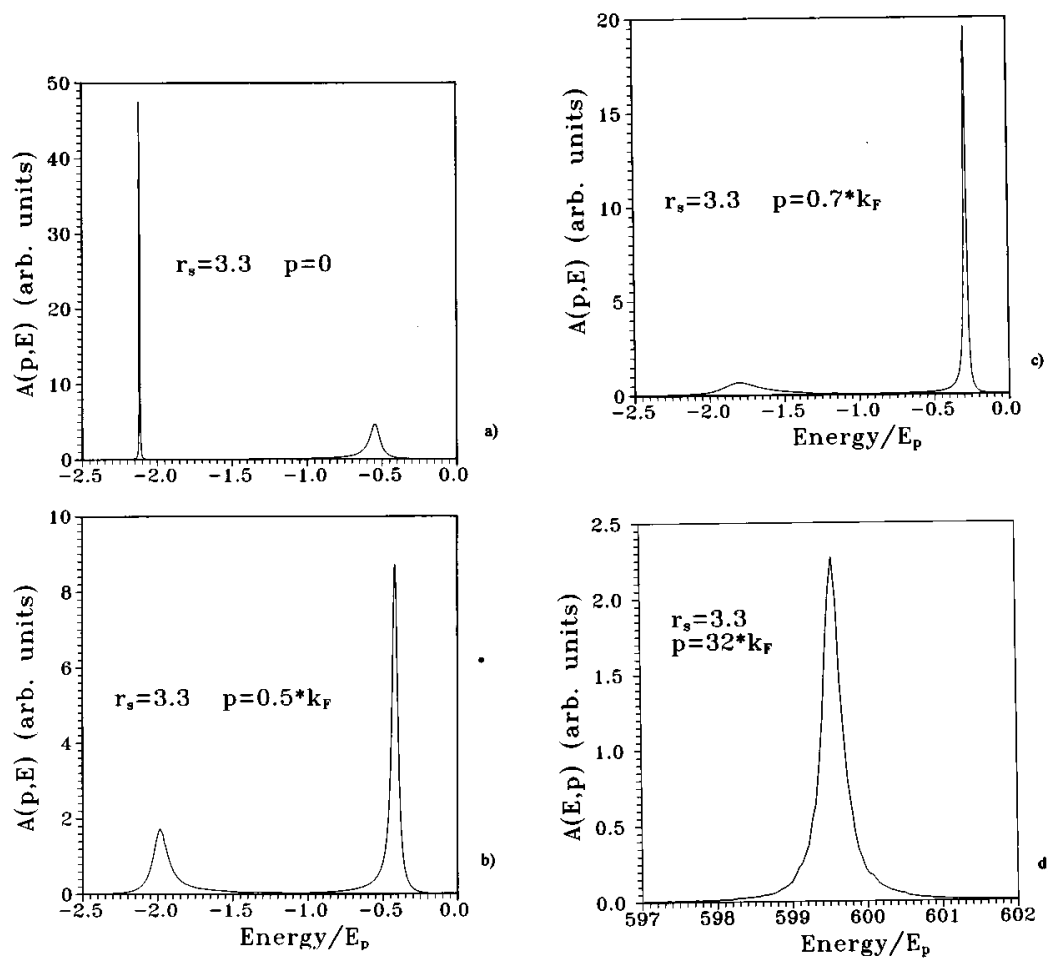

FIG. 11. Spectral density functions $A(p, E)$ of jellium $\left(r_{s}=3.3\right)$. (a) $p=0$. (b) $p=0.5 \times k_{F}$. (c) $p=0.7 k_{F}$. (d) $p=32.0 \times k_{F}$. leads to a reduction of the valence-electron Compton peak by $4 \%$, when compared with the correlation-corrected one. This reduction is just what we have found for the [110] Li profiles (see Fig. 2). Certainly the reduction for the other two principal directions, as found in the experiment, is somewhat larger, see Fig. 3. Nevertheless, we feel we are on the right track as far as the reason for these discrepancies is concerned.

Likewise the smaller contribution of the secondary Fermi surfaces to the directional Compton profiles in the experi-

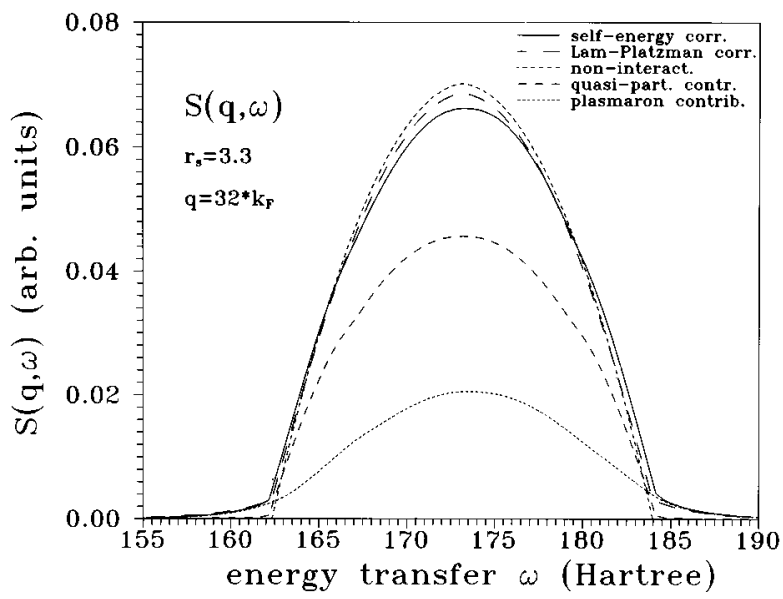

FIG. 12. Jellium $S(q, \omega)\left(r_{s}=3.3\right.$, corresponding to Li). For the assignment of the different line styles, see the inset. The upper three curves are as follows. Solid line: Self-energy corrected. Longdashed line: correlation corrected according to Ref. 20. Shortdashed line: free-electron inverted parabola, noninteracting. The lower two curves are as follows. Long-dashed line: contribution of the quasiparticle peak to the self-energy-corrected Compton profile. Short-dashed line: plasmaron-peak contribution to the self-energycorrected Compton profile. ment compared with the calculation finds its explanation, if one separately considers the contribution of the quasiparticle states to the higher-momentum components, on one hand, and the corresponding contribution of the plasmaron states on the other hand. For this reason we utilize Lundquist's ${ }^{47}$ lowest-order representation of the perturbed hole state, connected with the spectral weight function $A(\mathbf{k}, E)$ in Eq. (39), where the unperturbed hole state $c_{\mathbf{k}}|0\rangle$ is coupled to the product state $c_{\mathbf{k}-\mathbf{Q}} b_{-\mathbf{Q}}^{+}|0\rangle$, involving one hole and one plasmon, the plasmaron state. Let $|0\rangle$ be the filled Fermi sea, and $c_{\mathbf{k}}^{+}, c_{\mathbf{k}}$, and $b_{\mathbf{Q}}^{+}, b_{\mathbf{Q}}$ creation and annihilation operators for fermions and bosons (plasmons), respectively. Then we can write the perturbed hole state in the Bloch representation as follows:

$$
\begin{aligned}
\left|\psi_{\mathbf{k}, \nu}\right\rangle= & \left(1 / N_{\mathbf{k}, \nu}\right)\left\{c_{\mathbf{k}, \nu}+(\hbar / V)^{1 / 2}\right. \\
& \times \sum_{\mathbf{Q},|\mathbf{k}-\mathbf{Q}|<k_{F}} P g_{\mathbf{Q}}\left[c_{\mathbf{k}-\mathbf{Q}, \nu} b_{-\mathbf{Q}}^{+} /\left(E-E_{\nu}(\mathbf{k}-\mathbf{Q})\right.\right. \\
& \left.\left.\left.+\hbar \omega_{\mathbf{Q}}\right)\right]\right\}|0\rangle
\end{aligned}
$$

where $N_{\mathbf{k}}$ is a normalization factor, $P$ means that the principal value should be taken, $g_{\mathbf{Q}}$ is the electron-plasmon coupling strength, $E_{\nu}(\mathbf{k})$ is the single-particle eigenvalue spectrum, and $\hbar \omega_{\mathbf{Q}}$ is the energy of a plasmon with wave vector Q.

Assume that in a Compton scattering process the first term on the right-hand side of Eq. (40), $c_{\mathbf{k}, \nu}|0\rangle$, might give rise to higher-momentum components (HMC's) due to umklapp processes at k, so that these HMC's strongly contribute to directional differences of Compton profiles. Then the second term on the right side of Eq. (40) (plasmaron states) 
will diminish the amplitude of the directional differences, since the $\mathbf{Q}$ summation makes the contributions of HMC's, due to umklapp processes at $\mathbf{k}-\mathbf{Q}$, isotropically distributed. LDA does not take into account the self-energy-induced plasmaron states. Therefore, the LDA-calculated directional Compton profile differences must be reduced, when selfenergy effects are properly allowed for. According to the jellium calculations, presented in Fig. 12, the contribution of the second (isotropic) term of Eq. (40) to the momentum density is roughly $30 \%$. Therefore this isotropic contribution can, at least partly, account for the discrepancy between measured and LDA-calculated Compton profile anisotropies. As already discussed in Sec. IV C, this contribution could even be larger in a real metallic solid due to additional collective states (so-called zone-boundary collective states).

\section{CONCLUSIONS}

(1) All measured total directional valence-electron Compton profiles of $\mathrm{Li}$ exhibit smaller values at $p_{z}=0$, and larger values at $p_{z} \approx k_{F}$ than the corresponding LDA-calculated ones, where the latter are convoluted with the experimental resolution, and correlation corrected according to Lam and Platzman. ${ }^{20}$ The q-orientation dependence of this discrepancy is consistent with a contribution of the secondary Fermi surfaces to the momentum density, which is smaller than predicted by the LDA calculations. This leads to oscillations of the measured directional differences with amplitudes smaller than the LDA-calculated ones.

(2) The above-mentioned discrepancies can be interpreted as being due to self-energy effects connected with the Compton-scattering process. The double-peak structure of the spectral density function, which describes the creation of the hole during the scattering process, leads to two Compton profiles separated on an energy scale by 1.5 times the plasmon energy, where the second peak of the spectral density function is attributed to the so-called plasmaron, a bound plasmon-hole state. This doubling of the Compton profiles can account, even quantitatively, for the lower maximum value of the resulting profile, when compared with a theoretical one, calculated within the limits of the LDA, on the basis of the impulse approximation. Moreover, it could be shown that the formation of the plasmaron state during the Compton-scattering process diminishes the contribution of higher-momentum components, or will lead to their smearing over momentum space, which can account for the reduced oscillations of the measured orientation difference profiles when compared with the LDA-calculated ones.

(3) The reconstructed 3D momentum density exhibits higher-momentum components due to the umklapp processes near the 110 zone boundary, visible in the anisotropic part of the reconstructed momentum density

(4) The reconstructed 3D occupation number density enables a determination of the Fermi-surface anisotropy, after subtraction of a systematic error. The maximum Fermisurface anisotropy (relative difference between the diameter of the Fermi surface in [110] and [100] directions) was found to be $3.6 \pm 1.1 \%$, where this value is closer to the result of theories with a nonlocal treatment of electron correlation than to findings of "local" theories.

(5) The shape of the occupation number density function of Li near the Fermi momentum in the [100] direction, as found by means of a 3D reconstruction method, fits a jellium model, subjected to the same reconstruction procedure, for $z=0.1 \pm 0.1$, where $z$ is the renormalization factor. This finding can be interpreted either as being due to a drastic reduction of $z$ in a real metallic solid, as being brought about by additional channels for collective excitations, which lets us increase the plasmaron contribution, or as a hint that the nondiagonal elements of the occupation number density, mixing between different bands, cannot be neglected.

\section{ACKNOWLEDGMENTS}

We would like to thank A. Bansil and S. Kaprzyk for providing result of their LDA calculations prior to publication, and for many fruitful conversations. Helpful discussions with N. Shiotani are grateful acknowledged. We thank K.-J. Gabriel for help with measurements. This work was funded by the German Federal Ministry of Research and Technology under Contract No. 05 5PEAXB 6.

\section{APPENDIX A}

Let $I_{i}$ be the distribution of the number of scattered photons in a Compton experiment, measured on an equidistant $p_{z}$ scale (distance $\Delta p_{z}$ ). We assume the measurement at different points to be uncorrelated, and to be subjected to Poisson statistics. Then

$$
\operatorname{cov}\left(I_{i}, I_{j}\right)=I_{i} \delta_{i j}
$$

The following relation between $I_{i}$ and the Compton profile $J\left(p_{z i}\right)$ holds:

$$
J\left(p_{z i}\right)=\left(Z / N \Delta p_{z}\right) I_{i},
$$

where we assume the Compton profile to be normalized to the number $Z$ of electrons per atom, and $N=\Sigma_{i} I_{i}$. For the covariance $^{55}$ of $J\left(p_{z}\right)$ we obtain

$$
\begin{aligned}
\operatorname{cov}\left(J\left(p_{z i}\right), J\left(p_{z j}\right)\right)= & \sum_{n} \sum_{m}\left(\partial J\left(p_{z i}\right) / \partial I_{n}\right)\left(\partial J\left(p_{z j}\right) / \partial I_{m}\right) \\
& \times \operatorname{cov}\left(I_{n}, I_{m}\right) \\
= & \left(Z / N \Delta p_{z}\right) J\left(p_{z i}\right) \delta_{i j}-J\left(p_{z i}\right) J\left(p_{z j}\right) .
\end{aligned}
$$

According to Eq. (11), and taking into account the symmetry of the Compton profile, we can write the reciprocal form factor as a discrete cosinus transform:

$$
B(z)=\sum_{i} J\left(p_{z i}\right) \cos \left(p_{z i} z\right) \Delta p_{z}
$$

Using Eq. (A3), we can write the covariance of $B(z)$ in the following way: 


$$
\begin{aligned}
\operatorname{cov}\left(B\left(z_{i}\right), B\left(z_{j}\right)\right)= & \left(Z^{2} / 2 N\right)\left\{\left[B\left(z_{i}+z_{j}\right) / Z\right]+\left[B\left(z_{i}-z_{j}\right) / Z\right]\right. \\
& \left.-2\left[B\left(z_{i}\right) B\left(z_{j}\right) / Z^{2}\right]\right\} \\
\approx & \left\{\begin{array}{lll}
0 & \text { for } z_{i}=0 & \text { or } \\
(Z / 2 N) B\left(z_{i}-z_{j}\right) & \text { else, }
\end{array}\right.
\end{aligned}
$$

where the approximation uses the fact that $B(z)$ is a rapidly decreasing function of $z$.

Relations (A3) and (A5) apply not only to the total Compton profile and its $B$ function but also to the valence part, since the subtracted core profile can be assumed to be free of error. According to Eq. (15), the expansion coefficients $b_{l}$ are obtained by solving a system of $M$ linear equations, where $M$ is the number of different crystal orientations. Since the anisotropy of $B(z)$ is small compared to $B(0)$, we can approximate the covariance of $B(z)$ to be independent of the crystal orientation. If we additionally assume that the measurements of the Compton profiles with different orientation of the momentum transfer are performed independently, we can write

$$
\operatorname{cov}\left(B_{r}\left(r_{i}\right), B_{s}\left(r_{j}\right)\right)=\delta_{r s} \operatorname{cov}\left(B_{r}\left(r_{i}\right), B_{r}\left(r_{j}\right)\right),
$$

where $r$ and $s$ refer to different crystal orientations. For practical calculations we can insert the orientation averaged $B(r)$ functions into Eq. (A6). By taking into account that there exists a twofold correlation for the expansion coefficients $b_{l}$, one ends up with

$$
\begin{aligned}
\operatorname{cov}\left(b_{k}\left(r_{i}\right), b_{l}\left(r_{j}\right)\right)= & \sum_{r} \sum_{s}\left[\partial b_{k}\left(r_{i}\right) / \partial B_{r}\left(r_{i}\right)\right] \\
& \times\left[\partial b_{l}\left(r_{j}\right) / \partial B_{s}\left(r_{j}\right)\right] \operatorname{cov}\left(B_{r}\left(r_{i}\right), B_{s}\left(r_{j}\right)\right) \\
= & \left(\mathbf{F}^{t} \mathbf{F}\right)_{k l}^{-1} \operatorname{cov}\left(B\left(r_{i}\right), B\left(r_{j}\right)\right) .
\end{aligned}
$$

We can approximate the integral of Eq. (17) by a discrete summation

$$
\rho_{l}(p)=i^{l}\left(1 / 2 \pi^{2}\right) \sum_{i} b_{l}\left(r_{i}\right) j_{l}\left(p r_{i}\right) r_{i}^{2} \Delta r,
$$

so that the covariance of the expansion coefficients of the momentum density, $\rho_{l}(p)$, can be written (taking again into account that they also are twofold correlated)

$$
\begin{aligned}
\operatorname{cov}\left(\rho_{k}\left(p_{i}\right), \rho_{l}\left(p_{j}\right)\right) \\
=\sum_{m} \sum_{n}\left[\partial \rho_{k}\left(p_{i}\right) / \partial b_{k}\left(r_{m}\right)\right]\left[\partial \rho_{l}\left(p_{j}\right) / \partial b_{l}\left(r_{n}\right)\right] \\
\quad \times \operatorname{cov}\left(b_{k}\left(r_{m}\right), b_{l}\left(r_{n}\right)\right) \\
=i^{k+l}\left(1 / 4 \pi^{4}\right)\left(\mathbf{F}^{t} \mathbf{F}\right)_{k l}^{-1} \sum_{m} \sum_{n} j_{k}\left(p_{i} r_{m}\right) r_{m}^{2} \Delta r \\
\quad \times j_{l}\left(p_{j} r_{n}\right) r_{m}^{2} \Delta r \operatorname{cov}\left(B\left(r_{m}\right), B\left(r_{n}\right)\right) .
\end{aligned}
$$

Thus by using Eq. (A9) together with Eq. (A5) the variance of the 3D-reconstructed momentum density $\rho(\mathbf{p})$ can be calculated:

$$
\sigma_{\rho}^{2}(\mathbf{p})=\sum_{k} \sum_{l} F_{k}\left(\Omega_{\mathbf{p}}\right) F_{l}\left(\Omega_{\mathbf{p}}\right) \operatorname{cov}\left(\rho_{k}(p), \rho_{l}(p)\right) .
$$

\section{APPENDIX B}

Equation (12) enables us to obtain the reciprocal form factor $B(\mathbf{r})$ for any $\mathbf{r}$, provided the expansion coefficients $b_{l}(r)$ are known. Using Eq. (12) together with Eq. (A7), we can calculate the covariance of $B(\mathbf{R})$, where $\mathbf{R}$ is a lattice translational vector:

$$
\begin{aligned}
\operatorname{cov}\left(B(\mathbf{R}), B\left(\mathbf{R}^{\prime}\right)\right)= & \operatorname{cov}\left(B(R), B\left(R^{\prime}\right)\right) \\
& \times \sum_{k} \sum_{l} F_{k}\left(\Omega_{\mathbf{R}}\right) F_{l}\left(\Omega_{\mathbf{R}^{\prime}}\right)\left(\mathbf{F}^{t} \mathbf{F}\right)_{k l}^{-1} .
\end{aligned}
$$

By means of Eq. (20) and using Eq. (B1), we end up with the following expression for the variance of the occupation number density $N(\mathbf{k})$ :

$$
\begin{aligned}
\sigma_{N}^{2}(\mathbf{k})= & \sum_{\mathbf{R}} \sum_{\mathbf{R}^{\prime}} \exp \left[i \mathbf{k} \cdot\left(\mathbf{R}+\mathbf{R}^{\prime}\right)\right] \operatorname{cov}\left(B(R), B\left(R^{\prime}\right)\right) \\
& \times \sum_{k} \sum_{l} F_{k}\left(\Omega_{\mathbf{R}}\right) F_{l}\left(\Omega_{\mathbf{R}^{\prime}}\right)\left(\mathbf{F}^{t} \mathbf{F}\right)_{k l}^{-1} .
\end{aligned}
$$

\section{APPENDIX C}

The electron density in position space, $\rho(\mathbf{r})$, is expressed in terms of field operators

$$
\rho(\mathbf{r})=\left\langle\Psi^{+}(\mathbf{r}, 0) \Psi(\mathbf{r}, 0)\right\rangle .
$$

By inserting both the expansion of the field operators in Bloch waves, according to Eq. (8), and the expansion of the Bloch waves in plane waves [Eq. (9)], one ends up with

$$
\begin{aligned}
\rho(\mathbf{r})= & V^{-1} \sum_{\nu \nu^{\prime}} \sum_{k k^{\prime}}\left\langle a_{\mathbf{k}^{\prime}, \nu^{\prime}}^{+}(0) a_{\mathbf{k}, \nu}(0)\right\rangle \sum_{\mathbf{G G}^{\prime}} \alpha_{\nu^{\prime}}^{*}\left(\mathbf{k}^{\prime}+\mathbf{G}^{\prime}\right) \alpha_{\nu}(\mathbf{k} \\
& +\mathbf{G}) \times \exp \left[i\left(\mathbf{k}^{\prime}+\mathbf{G}^{\prime}-\mathbf{k}-\mathbf{G}\right) \cdot \mathbf{r}\right] .
\end{aligned}
$$

The spatial average

$$
\bar{\rho} \equiv V^{-1} \int \rho(\mathbf{r}) d \mathbf{r}
$$

of the electron density is then given by 


$$
\begin{aligned}
\bar{\rho}= & V^{-1} \sum_{\nu \nu^{\prime}} \sum_{\mathbf{k} \mathbf{k}^{\prime}}\left\langle a_{\mathbf{k}^{\prime}, \nu^{\prime}}^{+}(0) a_{\mathbf{k}, \nu}(0)\right\rangle \sum_{\mathbf{G G}^{\prime}} \alpha_{\nu^{\prime}}^{*}\left(\mathbf{k}^{\prime}+\mathbf{G}^{\prime}\right) \alpha_{\nu}(\mathbf{k} \\
& +\mathbf{G}) \delta\left(\mathbf{k}-\mathbf{k}^{\prime}\right) \delta\left(\mathbf{G}-\mathbf{G}^{\prime}\right) \\
= & V^{-1} \sum_{\mathbf{k}} \sum_{\nu \nu^{\prime}} n_{\nu \nu^{\prime}}(\mathbf{k}) \sum_{\mathbf{G}} \alpha_{\nu^{\prime}}^{*}(\mathbf{k}+\mathbf{G}) \alpha_{\nu}(\mathbf{k}+\mathbf{G}) \equiv \sum_{\mathbf{k}} \overline{\rho_{\mathbf{k}}} .
\end{aligned}
$$

Now we can easily express $B(\mathbf{R})$ of Eq. (21) in terms of the kth contribution $\overline{\rho_{\mathbf{k}}}$ to the spatial average of the electron density, namely,

$$
B(\mathbf{R})=8 \pi^{3} \sum_{\mathbf{k}} \overline{\rho_{\mathbf{k}}} \exp (i \mathbf{k} \cdot \mathbf{R})
$$

which is just the relation of Eq. (24).
${ }^{1}$ J. W. M. DuMond, Phys. Rev. 33, 643 (1929).

${ }^{2}$ P. Eisenberger and P. M. Platzman, Phys. Rev. A 2, 415 (1970).

${ }^{3}$ M. J. Cooper, J. A. Leake, and R. J. Weiss, Philos. Mag. 12, 797 (1965).

${ }^{4}$ P. Eisenberger, L. Lam, P. M. Platzman, and P. Schmidt, Phys. Rev. B 6, 3671 (1972).

${ }^{5}$ P. Eisenberger and W. A. Reed, Phys. Rev. B 9, 3237 (1974).

${ }^{6}$ M. J. Cooper, Rep. Prog. Phys. 48, 415 (1985).

${ }^{7}$ W. Schülke, Phys. Status Solidi B 82, 229 (1977).

${ }^{8}$ P. Pattison, W. Weyrich, and B. G. Williams, Solid State Commun. 21, 967 (1977).

${ }^{9}$ W. Weyrich, P. Pattison, and B. G. Williams, Chem. Phys. 41, 271 (1979).

${ }^{10}$ N. K. Hansen, Reconstruction of the EMD from a Set of Directional Compton Profiles (Hahn Meitner Institute, Berlin, 1980), Rep. HMI B 342.

${ }^{11}$ N. K. Hansen, P. Pattison, and J. R. Schneider, Z. Phys. B 66, 305 (1987)

${ }^{12}$ W. Schülke, Phys. Status Solidi B 80, K67 (1977).

${ }^{13}$ W. Schülke, Jpn. J. Appl. Phys. 17, 332 (1978).

${ }^{14}$ G. Loupias and J. Petiau, J. Phys. (Paris) 41, 265 (1980).

${ }^{15}$ N. Shiotani, N Sakai, F. Itoh, M. Sakurai, H. Kawata, Y. Amemiya, and M. Ando, Nucl. Instrum. Methods Phys. Res. Sect. A 275, 447 (1989).

${ }^{16}$ A. Berthold, S. Mourikis, J. R. Schmitz, W. Schülke, and H. Schulte-Schrepping, Nucl. Instrum. Methods Phys. Res. Sect. A 317, 373 (1992).

${ }^{17}$ G. Loupias, J. Petiau, A. Issolah, and M. Schneider, Phys. Status Solidi B 102, 79 (1980).

${ }^{18}$ Y. Sakurai, V. Tanaka, A. Bansil, S. Kaprzyk, A. T. Stewart, Y. Nagashima, T. Hyodo, S. Nanao, H. Kawata and N. Shiotani, Phys. Rev. Lett. 74, 2252 (1995). Reference 18 presents LDAcalculated Compton profiles only for the three principal directions. LDA-calculated Compton profiles for the other eight directions measured in our experiment were kindly provided by A. Bansil and S. Kaprzyk prior to publication.

${ }^{19}$ C. Blaas, J. Redinger, S. Manninen, V. Honkimäki, K. Hämäläinen, and P. Suortti, Phys. Rev. Lett. 75, 1984 (1995).

${ }^{20}$ L. Lam and P. M. Platzman, Phys. Rev. B 9, 5122 (1974).

${ }^{21}$ P. Nozieres and D. Pines, Phys. Rev. 113, 1254 (1959).

${ }^{22}$ R. Ribberfors, Phys. Rev. B 12, 3136 (1975).

${ }^{23}$ P. Holm and R. Ribberfors, Phys. Rev. A 40, 6251 (1989); A. Issolah, B. Levy, A. Beswick, and G. Loupias, ibid. 38, 4509 (1988).

${ }^{24}$ E. Daniel and S. H. Vosko, Phys. Rev. 120, 2041 (1960).

${ }^{25}$ B. I. Lundquist and C. Lyden, Phys. Rev. B 4, 3360 (1971).
${ }^{26}$ Since we will use only the diagonal terms of $n_{\nu \nu^{\prime}}(\mathbf{k})$ in Eq. (8), we will skip the double summation $\nu, \nu^{\prime}$ in what follows.

${ }^{27}$ F. M. Müller and M. G. Priestley, Phys. Rev. 148, 638 (1966).

${ }^{28}$ D. G. Lock, V. H. C. Crisp, and R. N. West, J. Phys. F 3, 561 (1973).

${ }^{29}$ A. Berthold, J. Degenhardt, S. Mourikis, J. R. Schmitz, W. Schülke, H. Schulte-Schrepping, F. Wohlert, A. Hamacher, D. Protic, and G. Riepe, Nucl. Instrum. Methods Phys. Res. Sect. A 320, 375 (1992).

${ }^{30}$ W. Schülke, in Handbook on Synchrotron Radiation, edited by G. Brown and D. E. Moncton (North-Holland, Amsterdam, 1991), Vol. 3, p. 609.

${ }^{31}$ N. Sakai, J. Phys. Soc. Jpn. 56, 2477 (1987).

${ }^{32}$ P. Paatero and V. Halonen, Nucl. Instrum. Methods 135, 537 (1976).

${ }^{33}$ F. Biggs, L. B. Mendelsohn, and J. B. Mann, At. Data Nucl. Data Tables 16, 201 (1975).

${ }^{34}$ L. Oberli, A. A. Manuel, R. Sachot, P. Descouts, and M. Peter, Phys. Rev. B 31, 6104 (1985).

${ }^{35}$ A. H. MacDonald, J. Phys. F 10, 1737 (1980).

${ }^{36}$ M. B. Hunt, P. H. Reinders, and M. Springford, J. Phys. Condens. Matter 1, 6589 (1989).

${ }^{37}$ D. L. Randles and M. Springford, J. Phys. F 6, 1827 (1976).

${ }^{38}$ J. J. Donaghy and A. T. Stewart, Phys. Rev. 164, 391 (1967).

${ }^{39}$ J. J. Paciga and D. Williams, Can. J. Phys. 49, 3227 (1971).

${ }^{40}$ S. S. Rajput, P. Prasad, R. M. Singru, W. Triftshäuser, A. Eckert, G. Kögel, S. Kaprzyk, and A. Bansil, J. Phys. Condens. Matter 5, 6419 (1993).

${ }^{41}$ M. Rasolt, S. B. Nickerson, and S. H. Vosko, Solid State Commun. 16, 827 (1975).

${ }^{42}$ J. Lam, Phys. Rev. B 3, 3243 (1971).

${ }^{43}$ A. W. Overhauser, Phys. Rev. B 3, 1888 (1971).

${ }^{44}$ L. J. Lantto, Phys. Rev. B 22, 1380 (1980).

${ }^{45}$ Y. Takada and H. Yasuhara, Phys. Rev. B 44, 7879 (1991).

${ }^{46}$ D. M. Ceperley and B. J. Adler, Phys. Rev. Lett. 45, 566 (1980).

${ }^{47}$ B. I. Lundquist, Phys. Konden. Mater. 7, 117 (1968).

${ }^{48}$ E.-Ni Foo and J. J. Hopfield, Phys. Rev. 173, 635 (1968).

${ }^{49}$ K. Sturm and L. E. Oliviera, Phys. Rev. B 30, 4351 (1984).

${ }^{50}$ W. Schülke, H. Nagasawa, S. Mourikis, and P. Lanzki, Phys. Rev. B 33, 6744 (1986).

${ }^{51}$ W. Schülke, H. Nagasawa, S. Mourikis, and A. Kaprolat, Phys. Rev. B 40, 12215 (1989).

${ }^{52}$ T. K. Ng and B. Dabrowski, Phys. Rev. B 33, 5358 (1986).

${ }^{53}$ B. I. Lundquist, Phys. Status Solidi 32, 273 (1969).

${ }^{54}$ K. Hämäläinen (private communication).

${ }^{55}$ R. J. Barlow, Statistics (Wiley, Chichester, 1989). 